\title{
Competing Spin Liquids and Hidden Spin-Nematic Order in Spin Ice with Frustrated Transverse Exchange
}

\author{
Mathieu Taillefumier, ${ }^{1}$ Owen Benton, ${ }^{2}$ Han Yan, ${ }^{1}$ L. D. C. Jaubert, ${ }^{3}$ and Nic Shannon ${ }^{1}$ \\ ${ }^{1}$ Okinawa Institute of Science and Technology Graduate University, Onna-son, Okinawa 904-0495, Japan \\ ${ }^{2}$ RIKEN Center for Emergent Matter Science (CEMS), Wako, Saitama 351-0198, Japan \\ ${ }^{3}$ CNRS, Université de Bordeaux, LOMA, UMR 5798, 33400 Talence, France
}

(Received 29 April 2017; revised manuscript received 23 October 2017; published 6 December 2017)

Frustration in magnetic interactions can give rise to disordered ground states with subtle and beautiful properties. The spin ices $\mathrm{Ho}_{2} \mathrm{Ti}_{2} \mathrm{O}_{7}$ and $\mathrm{Dy}_{2} \mathrm{Ti}_{2} \mathrm{O}_{7}$ exemplify this phenomenon, displaying a classical spinliquid state, with fractionalized magnetic-monopole excitations. Recently, there has been great interest in closely related "quantum spin-ice" materials, following the realization that anisotropic exchange interactions could convert spin ice into a massively entangled, quantum spin liquid, where magnetic monopoles become the charges of an emergent quantum electrodynamics. Here we show that even the simplest model of a quantum spin ice, the XXZ model on the pyrochlore lattice, can realize a still-richer scenario. Using a combination of classical Monte Carlo simulation, semiclassical molecular-dynamics simulation, and analytic field theory, we explore the properties of this model for frustrated transverse exchange. We find not one, but three competing forms of spin liquid, as well as a phase with hidden, spin-nematic order. We explore the experimental signatures of each of these different states, making explicit predictions for inelastic neutron scattering. These results show an intriguing similarity to experiments on a range of pyrochlore oxides.

DOI: 10.1103/PhysRevX.7.041057

\section{INTRODUCTION}

The search for spin liquids-disordered phases of magnets that support entirely new forms of magnetic excitation-has become one of the defining themes of modern condensed-matter physics [1-3]. In this context, the pyrochlore lattice, a corner-sharing network of tetrahedra found in a wide range of naturally occurring minerals, has proved to be an amazing gift to science. Pyrochlore magnets play host to a variety of unconventional forms of magnetic order and include systems that have not been observed to order at any temperature [4]. Perhaps the most celebrated of these is the "spin ice" found in the Ising magnets $\mathrm{Ho}_{2} \mathrm{Ti}_{2} \mathrm{O}_{7}$ and $\mathrm{Dy}_{2} \mathrm{Ti}_{2} \mathrm{O}_{7}$ [5], a classical spin liquid described by an emergent $\mathrm{U}(1)$ lattice gauge theory with magnetic-monopole excitations [6].

As the understanding of spin ice has grown, so more attention has been given to the role of quantum effects. These are of particular relevance where a spin ice arises through anisotropic exchange interactions in a pyrochlore magnet [7-12] and have the potential to convert classical spin ice into a massively entangled, quantum spin liquid, described by an emergent U(1) quantum electrodynamics [12-25]. At the same time, great progress has been made in

Published by the American Physical Society under the terms of the Creative Commons Attribution 4.0 International license. Further distribution of this work must maintain attribution to the author(s) and the published article's title, journal citation, and DOI.
Subject Areas: Condensed Matter Physics,

Magnetism, Strongly Correlated Materials synthesizing and characterizing magnetic pyrochlore oxides. As well as revealing a number of candidates for quantum spin-ice behavior [26-34], these experiments have turned up many unusual and unexpected magnetic states in systems with strongly anisotropic exchange [35-45].

The main message of this article is that even the simplest model of a quantum spin ice-the XXZ model on a pyrochlore lattice - has far more to offer than spin ice alone. Working in the classical limit, accessible to large-scale simulation, we find that frustrated transverse exchange gives rise to not one, but three distinct spin-liquid regimes (Fig. 1). We explore the way in which these spin liquids compete, identify the different gauge groups associated with each spin liquid, and make explicit predictions for their experimental signatures (Fig. 2). We find that one of these spin liquids posses a highly unusual $\mathrm{U}(1) \times \mathrm{U}(1)$ gauge structure and, as an added bonus, undergoes a phase transition into a state with hidden, spin-nematic, order. We also use molecular dynamics simulations to characterize the excitations of this spinnematic phase (Fig. 5). The portrait that emerges has striking similarities to the behavior of a number of pyrochlore materials.

The simplest model able to capture quantum effects in a spin ice [7-12] is the XXZ model on the pyrochlore lattice

$$
\mathcal{H}_{\mathrm{XXZ}}=\sum_{\langle i j\rangle} J_{\mathrm{zz}} \mathrm{S}_{i}^{z} \mathrm{~S}_{j}^{z}-J_{ \pm}\left(\mathrm{S}_{i}^{+} \mathrm{S}_{j}^{-}+\mathrm{S}_{i}^{-} \mathrm{S}_{j}^{+}\right),
$$

where $\mathbf{S}_{i}=\left(\mathrm{S}_{i}^{x}, \mathrm{~S}_{i}^{y}, \mathrm{~S}_{i}^{z}\right)$ is a (pseudo-)spin-half operator describing the two states of the lowest-energy doublet of a 
(a) Finite-temperature phase diagram

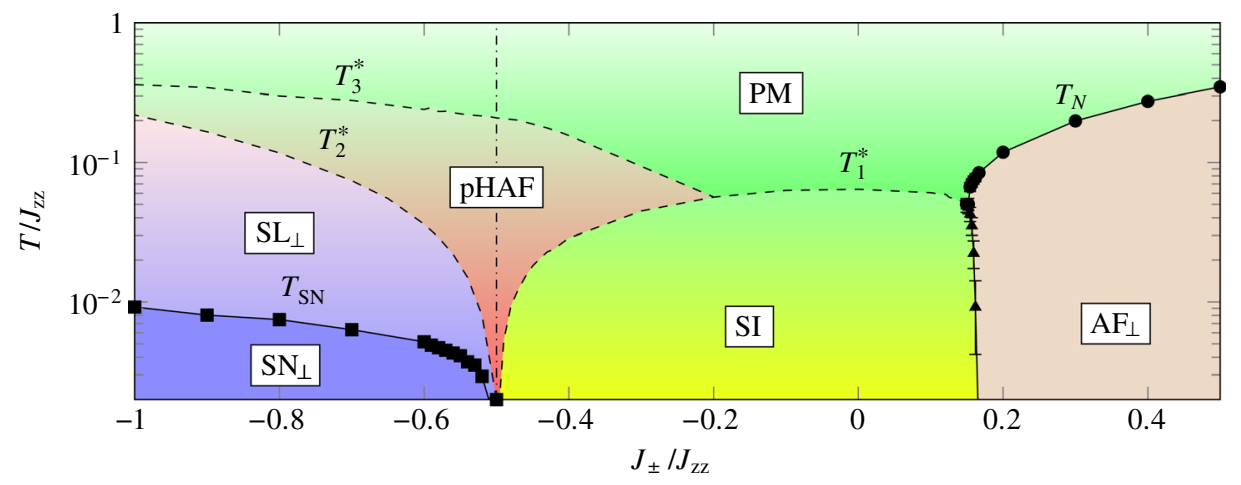

(b) Easy-plane spin-nematic

(c) Spin ice (SI)

(d) Easy-plane antiferromagnet

$\left(\mathrm{SN}_{\perp}\right)$
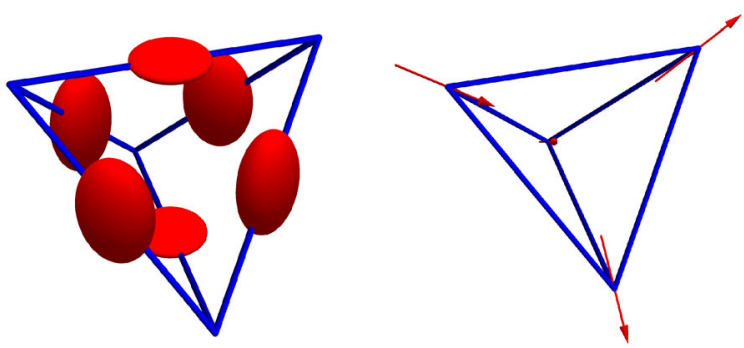

$\left(\mathrm{AF}_{\perp}\right)$

FIG. 1. (a) Finite-temperature phase diagram of the minimal model of a quantum spin ice, $\mathcal{H}_{\mathrm{XXZ}}$ [Eq. (1)], as found in classical Monte Carlo simulation. The model possesses three distinct spin-liquid regimes, spin ice (SI), an easy-plane spin liquid ( $\left.\mathrm{SL}_{\perp}\right)$, and the pseudoHeisenberg antiferromagnet $(\mathrm{pHAF})$, as well as phases with easy-plane antiferromagnetic $\left(\mathrm{AF}_{\perp}\right)$ and easy-plane spin-nematic $\left(\mathrm{SN}_{\perp}\right)$ order. Associated crossover (phase-transition) temperatures are indicated with dashed (solid) lines. For $J_{ \pm} / J_{\mathrm{zz}}=-1 / 2$ (dashed-dotted line), the model has an SU(2) symmetry and is thermodynamically equivalent to the Heisenberg antiferromagnet on a pyrochlore lattice. (b) Representation of bond quadrupolar order in the easy-plane spin-nematic phase ( $\mathrm{SN}_{\perp}$ ) (cf. Appendix A). $\mathrm{SN}_{\perp}$ breaks both the $U(1)$ spin rotational symmetry of Eq. (1) and the point-group symmetry of the lattice. (c) "Two-in, two-out" configuration of spins in the spin ice regime (SI). (d) Representative configuration of spin dipoles in the easy-plane antiferromagnet $\left(\mathrm{AF}_{\perp}\right)$.

(a) SI $\left(J_{ \pm}=0, T=0.05 J_{\mathrm{zz}}\right)$

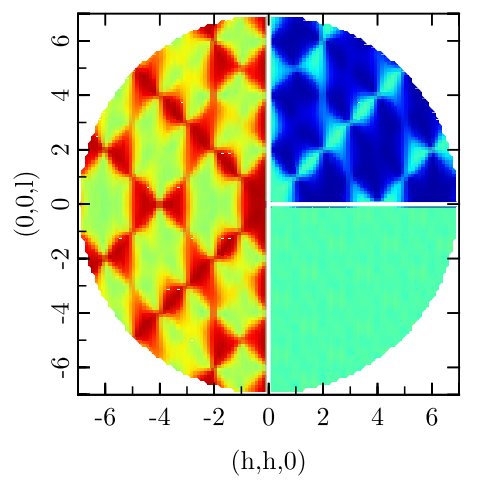

(b) $\mathrm{SN}_{\perp}, \mathrm{SL}_{\perp}\left(J_{ \pm}=-J_{\mathrm{zz}}, T=0.005 J_{\mathrm{zz}}\right)$.
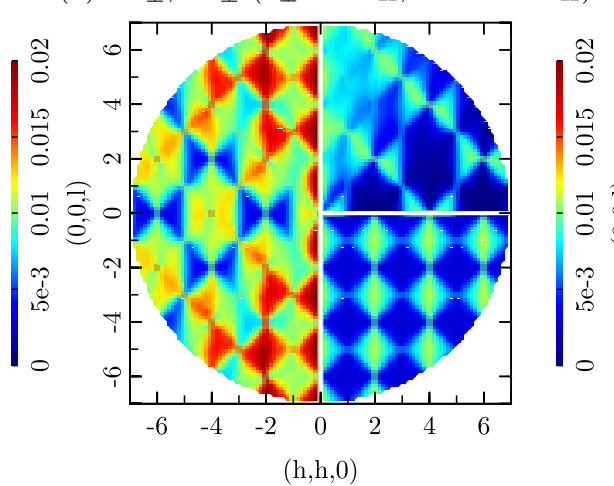

(c) $\operatorname{pHAF}\left(J_{ \pm}=-0.5 J_{\mathrm{zz}}, T=0.05 J_{\mathrm{zz}}\right)$

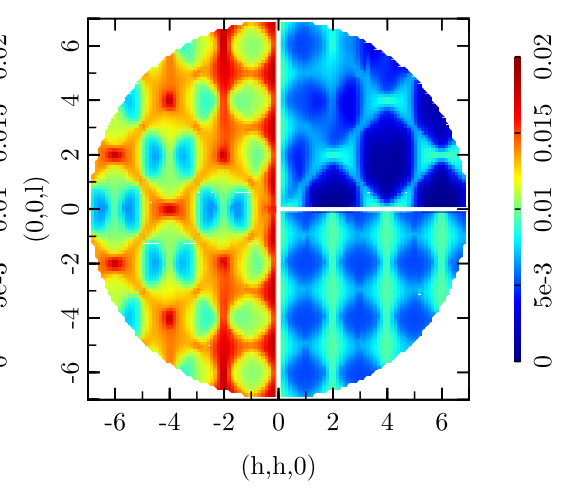

FIG. 2. Comparison of correlations in different spin-liquid and spin-nematic regimes. Left half of each panel: Equal-time structure factor $S(\mathbf{q})$, as measured in unpolarized neutron-scattering experiments. Right half of each panel: $S(\mathbf{q})$ resolved into spin-flip (SF, top) and non-spin-flip (NSF, bottom) components, as measured in polarized neutron-scattering experiments (cf. Ref. [46]). Definitions of these structure factors are given in Appendix C. (a) Spin ice (SI), showing "pinch points" indicative of algebraic spin correlations. (b) Phase with easy-plane spin-nematic order $\left(\mathrm{SN}_{\perp}\right)$, showing the absence of magnetic Bragg peaks and persistence of algebraic spin correlations. The same correlations are also observed in the easy-plane spin liquid $\left(\mathrm{SL}_{\perp}\right)$. (c) Spin liquid associated with the pseudoHeisenberg antiferromagnet (pHAF), showing algebraic spin correlations distinct from those in spin ice (SI) or the easy-plane spin liquid $\left(\mathrm{SL}_{\perp}\right)$. Results are taken from Monte Carlo simulations of $\mathcal{H}_{\mathrm{XXZ}}$ [Eq. (1)], for a cubic cluster of $N=8192$ spins. 
magnetic ion subject to a strong crystal electric field (CEF). The symmetry of the lattice requires the quantization axis of each spin (here, $\mathrm{S}_{i}^{z}$ ) to lie on a local [111] axis, as defined in Appendix A.

Ising interactions, $J_{\mathrm{zz}}>0$, favor states obeying the "ice rules" in which two spins point into and two spins point out of each tetrahedron on the lattice. The transverse term, $J_{ \pm}$, introduces dynamics about these spin-ice configurations and, for larger, positive values of $J_{ \pm} / J_{\mathrm{zz}}$, drives the system into a state with easy-plane order $[17,18,47-50]$. The physical meaning of this easy-plane order depends on the nature of the magnetic ion. For Kramers ions like $\mathrm{Yb}^{3+}$ and $\mathrm{Er}^{3+}$, all components of $\mathrm{S}$ relate to a magnetic dipole moment [11], and the ordered phase is an easy-plane antiferromagnet. However, for non-Kramers ions such as $\mathrm{Pr}^{3+}$ and $\mathrm{Tb}^{3+}[9,43]$ or "dipolar-octupolar" Kramers ions like $\mathrm{Nd}^{3+}$ or $\mathrm{Ce}^{3+}$ [51], the easy-plane order may have quadrupolar (octupolar) character. In what follows, we consider explicitly the case of Kramers ions. However, suitably reinterpreted, these results also have important implications for non-Kramers ions.

For $J_{ \pm}>0, \mathcal{H}_{\mathrm{XXZ}}$ [Eq. (1)] is unfrustrated, in the sense that it is free of sign problems in quantum Monte Carlo (QMC) simulation. In this case, the phase diagram is already well established [14,21]. For $J_{ \pm} / J_{\mathrm{zz}} \lesssim 0.05$, QMC simulations find a crossover from a conventional paramagnet into a classical spin liquid (spin ice) at a temperature $T^{*} / J_{\mathrm{zz}} \sim 0.2$ and a second crossover into a quantum spin liquid (QSL) at a much lower temperature $T_{\mathrm{QSL}}^{*} / J_{\mathrm{zz}} \sim$ $\left(J_{ \pm} / J_{\mathrm{zz}}\right)^{3}$. In the low-temperature quantum spin-liquid regime, the magnetic monopoles of classical spin ice become dynamic, fractional, spin excitations (spinons), while the spectrum of the model also includes gapless photons $[13,16]$. For $J_{ \pm} / J_{\mathrm{zz}} \gtrsim 0.05$, the U(1) QSL gives way to easy-plane antiferromagnetic order $\left(\mathrm{AF}_{\perp}\right)$, in which spins lie in the plane perpendicular to the local $\mathrm{S}^{z}$ axis [14,21].

Very little is known about the properties of $\mathcal{H}_{\mathrm{XXZ}}$ for $J_{ \pm}<0[10,18,43]$. On perturbative grounds, it is expected that the ground state for $\left|J_{ \pm}\right| / J_{\mathrm{zz}} \ll 1$ will also be a U(1) QSL [13], albeit one with a modified spinon dispersion [18,52]. Gauge mean-field calculations suggest that this QSL persists over a broad range of parameters, $-4.13 \lesssim$ $J_{ \pm} / J_{\mathrm{zz}}<0$ [18]. But the nature of competing ordered—or disordered-phases for $J_{ \pm}<0$ remains an open question.

There are many reasons to believe that the properties of the quantum spin-ice model, $\mathcal{H}_{\mathrm{XXZ}}$ [Eq. (1)] for frustrated coupling $J_{ \pm}<0$, could be even richer than for $J_{ \pm}>0$. In particular, for $J_{ \pm} / J_{\mathrm{zz}}=-\frac{1}{2}, \mathcal{H}_{\mathrm{XXZ}}$ [Eq. (1)] is equivalent (up to a site-dependent spin rotation), to the Heisenberg antiferromagnet (HAF) on a pyrochlore lattice. Like spin ice, the HAF is known to support a classical spin liquid [53-56], and it has also been argued to support a QSL ground state [57-60]. Crucially, both the classical and quantum spin liquids in the HAF have a qualitatively different character from those found in spin ice. This sets up a competition between two different kinds of spin liquid, namely, spin ice for $J_{ \pm} / J_{\mathrm{zz}} \approx 0$ and a state homologous to the HAF for $J_{ \pm} / J_{\mathrm{zz}} \approx-\frac{1}{2}$. It also opens the door for yet more novel magnetic phases for $J_{ \pm} / J_{\mathrm{zz}}<-\frac{1}{2}$.

\section{PHASE DIAGRAM DETERMINED BY CLASSICAL MONTE CARLO SIMULATIONS}

Since the quantum spin-ice model, $\mathcal{H}_{\mathrm{XXZ}}$ [Eq. (1)], is inaccessible to QMC for $J_{ \pm}<0$, we instead study its finitetemperature properties using classical Monte Carlo (MC) simulation - the results are summarized in the phase diagram Fig. 1. For $J_{ \pm}>0$, this phase diagram is very similar to that previously found in QMC simulations [14,21]—at a qualitative level, the only significant difference is the absence of a QSL below $T_{\mathrm{QSL}}^{*} / J_{\mathrm{zz}} \sim\left(J_{ \pm} / J_{\mathrm{zz}}\right)^{3} \lesssim 0.005$. At a quantitative level, we find changes in numerical values of the crossover temperature associated with the spin-ice regime, $T_{1}^{*}$, and the position of the zero-temperature boundary between $\mathrm{SI}$ and $\mathrm{AF}_{\perp}$. These changes can be ascribed to the fact that the magnetic monopoles (spinons) are not quantized in classical simulations and do not develop phase coherence [61]. Further details of classical MC simulations for $J_{ \pm}>0$ will be presented elsewhere [62].

We now turn to the frustrated case, $J_{ \pm}<0$. At low temperatures, spin-ice correlations persist up to $J_{ \pm} / J_{\mathrm{zz}}=$ $-\frac{1}{2}[10,43]$, as illustrated in Fig. 2(a). Upon reaching $J_{ \pm} / J_{\mathrm{zz}}=-\frac{1}{2}$, the system becomes thermodynamically equivalent to a HAF. This high-symmetry point gives rise to a new form of spin liquid at finite temperature, labeled a pseudo-Heisenberg antiferromagnet (pHAF) in Fig. 1. Once again, this spin liquid has algebraic correlations, as shown in Fig. 2(c), but with qualitatively different character from spin ice [Fig. 2(a)]. These correlations persist up to a crossover temperature $T_{3}^{*}$ associated with the Curie-law crossover (CLC) in the magnetic susceptibility [63].

While the correlations measured in the equal-time structure factor $S(\mathbf{q})$ are also different from those found in the HAF [54,55,64], the two models are equivalent up to a local coordinate transformation. By analogy with earlier work on the HAF $[55,56,65]$, the spin liquid pHAF can be described by a $\mathrm{U}(1) \times \mathrm{U}(1) \times \mathrm{U}(1)$ gauge theory.

The situation for $J_{ \pm} / J_{\mathrm{zz}}<-\frac{1}{2}$ is even more interesting. Below a second crossover scale, $T_{2}^{*}<T_{3}^{*}$, identifiable by a reduction in the fluctuations of the $z$-components of the spins (see Appendix B), the pHAF gives way to an easyplane spin liquid, labeled $\mathrm{SL}_{\perp}$ in Fig. 1. Spin correlations in this regime have algebraic character, with pinch points in $S(\mathbf{q})$ [Fig. 2(b)]. However these correlations are qualitatively different from those in either spin ice [Fig. 2(a)] or the pHAF [Fig. 2(c)]. At a still lower temperature, $T_{\mathrm{SN}}<T_{2}^{*}$, the system undergoes a thermodynamic phase transition, marked by a clear anomaly in the specific heat. Nonetheless, this phase transition does not give rise to any magnetic Bragg peaks in $S(\mathbf{q})$ and, at least as far as dipolar 
spin correlations are concerned, the system remains disordered.

While the new phase for $T<T_{\mathrm{SN}}$-labeled $\mathrm{SN}_{\perp}$ in Fig. 1-does not exhibit any conventional magnetic order, it does possess a hidden, spin-nematic order. The ordered state does not break translational symmetry, but breaks the $\mathrm{U}(1)$ symmetry of $\mathcal{H}_{\mathrm{XXZ}}$ [Eq. (1)] by selecting an axis within the local $x y$-plane. Such an order can be described by the bond-based order parameter [66-68]

$$
\mathbf{Q}_{\perp}=\sum_{\langle i j\rangle} \frac{1}{3 N}\left(\begin{array}{c}
\mathrm{S}_{i}^{x} \mathrm{~S}_{j}^{x}-\mathrm{S}_{i}^{y} \mathrm{~S}_{j}^{y} \\
\mathrm{~S}_{i}^{x} \mathrm{~S}_{j}^{y}+\mathrm{S}_{i}^{y} \mathrm{~S}_{j}^{x}
\end{array}\right),
$$

where the sum on $\langle i j\rangle$ runs over the nearest-neighbor bonds of the lattice, and $\mathrm{S}_{i}=\left(\mathrm{S}_{i}^{x}, \mathrm{~S}_{i}^{y}, \mathrm{~S}_{i}^{z}\right)$ is expressed in the local frame of site $i$ (cf. Appendix A).

This type of easy-plane order is formally identical to the spin-nematic phases found in a range of frustrated magnets in applied magnetic fields [68-70]. In common with these systems, the associated Landau theory

$$
F_{\mathrm{SN}_{\perp}}=a_{2}(T) \mathbf{Q}_{\perp}^{2}+a_{4} \mathbf{Q}_{\perp}^{4}+\cdots
$$

lacks a cubic term and therefore permits a continuous phase transition. Simulations suggest that the phase transition at $T=T_{\mathrm{SN}}$ is indeed continuous for $J_{ \pm} / J_{\mathrm{zz}} \lesssim-\frac{1}{2}$, becoming first order approaching the high-symmetry point $J_{ \pm} / J_{\mathrm{zz}} \rightarrow-\frac{1}{2}$. Further details of the thermodynamics of this transition are given in Appendix B.

\section{THEORY OF THE EASY-PLANE SPIN LIQUID}

Spin correlations in spin ice (SI) can be described using a $\mathrm{U}(1)$ lattice gauge theory [6,56,71], which gives rise to characteristic "pinch points" in the spin structure factor $S(\mathbf{q})$ [Fig. 2(a)]. Meanwhile, for classical spins, spin correlations in the Heisenberg AF on the pyrochlore lattice-and by extension in the $\mathrm{pHAF}$ - can be described using a $\mathrm{U}(1) \times$ $\mathrm{U}(1) \times \mathrm{U}(1)$ gauge theory [53-56]. The pHAF has qualitatively different pinch points from spin ice, as illustrated in Fig. 2(c). It is clear that the correlations of the easy-plane spin liquid, $\mathrm{SL}_{\perp}$ [Fig. 2(b)], are very different from either spin ice [Fig. 2(a)] or the pHAF [Fig. 2(c)]. Nonetheless, the presence of pinch points suggests that $\mathrm{SL}_{\perp}$, too, may be described by some form of gauge theory.

We can develop a field theory for the spin liquid $\mathrm{SL}_{\perp}$ by applying the methods developed in Refs. [50,72]. The starting point of this approach is to recast the spins $S_{i}$ in $\mathcal{H}_{\mathrm{XXZ}}$ [Eq. (1)] in terms of five order-parameter fields

$$
\left\{\mathbf{m}_{\lambda}\right\}=\left\{m_{\mathrm{A}_{2}}, \mathbf{m}_{\mathrm{E}}, \mathbf{m}_{\mathrm{T}_{1} \text { ice }}, \mathbf{m}_{\mathrm{T}_{1} \text { planar }}, \mathbf{m}_{\mathrm{T}_{2}}\right\}
$$

defined on each tetrahedron $\mathbf{r}$. These objects $\mathbf{m}_{\lambda}(\mathbf{r})$ describe the different kinds of four-sublattice magnetic orders consistent with the point-group symmetry of the pyrochlore lattice. Definitions of each field $\mathbf{m}_{\lambda}$ in terms of the spins $S_{i}$ are given in Appendix D.
The most general exchange Hamiltonian on the pyrochlore lattice can be transcribed exactly in terms of $\mathbf{m}_{\lambda}$ [49]. This greatly simplifies the determination of classical ground states and, where classical ground states form an extensive manifold, one can use this approach to determine the local constraints that control the resulting spin liquid $[50,72]$. In the case of $\mathrm{SL}_{\perp}$, for $T \rightarrow 0$, we have

$$
m_{\mathrm{A}_{2}}(\mathbf{r})=0, \quad \mathbf{m}_{\mathrm{E}}(\mathbf{r})=0, \quad \mathbf{m}_{\mathrm{T}_{1} \text { ice }}(\mathbf{r})=0 \quad \forall \mathbf{r} .
$$

The spin fluctuations at low temperature are thus dominated by the fluctuations of the remaining fields $\mathbf{m}_{\mathrm{T}_{2}}(\mathbf{r})$ and $\mathbf{m}_{\mathrm{T}_{1} \text { planar }}(\mathbf{r})$. These fields have significance as the order parameters of the competing four-sublattice magnetic orders that would be induced by the symmetryallowed perturbation

$$
\delta \mathcal{H}_{ \pm \pm}=\sum_{\langle i j\rangle} J_{ \pm \pm}\left[\gamma_{i j} \mathrm{~S}_{i}^{+} \mathbf{S}_{j}^{+}+\gamma_{i j}^{*} \mathrm{~S}_{i}^{-} \mathbf{S}_{j}^{-}\right],
$$

where $\gamma_{i j}$ are complex phase factors arising from the change in coordinate frame between different lattice sites [7,9-11,47,73]. For this reason, the spin liquid $\mathrm{SL}_{\perp}$ falls very naturally into the "multiple-phase competition" scenario for pyrochlore magnets $[49,50,74,75]$.

In Fig. 3, we show the classical ground-state phase diagram of the anisotropic exchange model

$$
\mathcal{H}_{\mathrm{ex}}=\mathcal{H}_{\mathrm{XXZ}}+\delta \mathcal{H}_{ \pm \pm}
$$

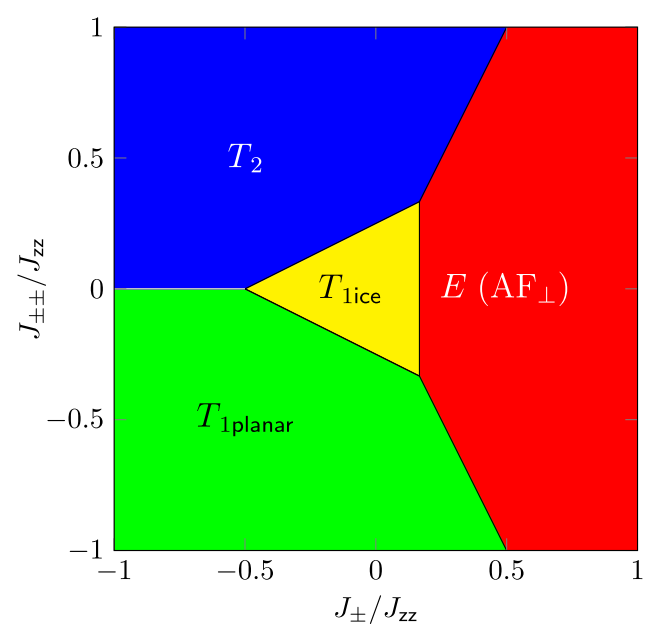

FIG. 3. Classical ground-state phase diagram of the anisotropic exchange model $\mathcal{H}_{\text {ex }}$ [Eq. (7)], for $J_{\mathrm{zz}}>0$. Different phases are labeled in terms of the irreps of the tetrahedral symmetry group, $T_{d}$ [Eq. (4)], as described in Ref. [49]. The minimal model of a quantum spin ice $\mathcal{H}_{X X Z}$ [Eq. (1)] exists on the line $J_{ \pm \pm}=0$-for $J_{ \pm}<-\frac{1}{2}$ (white line), two phases with four-sublattice easy-plane order meet, and the resulting enlarged ground-state manifold gives rise to the easy-plane spin liquid $\mathrm{SL}_{\perp}$ and spin-nematic phase $\mathrm{SN}_{\perp}$. A closely related mean-field phase diagram for nonKramers ions is given in Refs. [10,43]. 
This contains three distinct regions of four-sublattice order: the easy-plane ordered phases described by the fields $\mathbf{m}_{\mathrm{E}}$

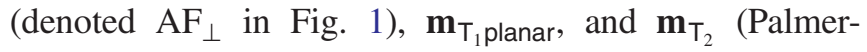
Chalker state [76]). These border a region of spin ice (denoted SI in Fig. 1) dominated by fluctuations of $\mathbf{m}_{\mathrm{T}_{1} \text { ice }}$. We note that a closely related phase diagram has been derived for non-Kramers ions [10,43]; in this case, easyplane order must be interpreted in terms of the quadrupole moment of the magnetic ion.

The nontrivial correlations in the spin liquid $\mathrm{SL}_{\perp}$ arise from the fact that neighboring tetrahedra share a spin, so that the fields $\mathbf{m}_{\lambda}(\mathbf{r})$ on neighboring tetrahedra are not independent of one another. This point, combined with Eq. (5), imposes spatial constraints on the fluctuations of $\mathbf{m}_{\mathrm{T}_{2}}(\mathbf{r})$ and $\mathbf{m}_{\mathrm{T}_{1} \text { planar }}(\mathbf{r})$. After coarse graining to extract the long wavelength physics, these constraints may be written in terms of two independent vector fluxes,

$$
\begin{aligned}
\mathbf{B}_{1}= & \frac{1}{2}\left(2 m_{\mathrm{T}_{1} \text { planar }}^{x},-\sqrt{3} m_{\mathrm{T}_{2}}^{y}-m_{\mathrm{T}_{1} \text { planar }}^{y}, \sqrt{3} m_{\mathrm{T}_{2}}^{z}-m_{\mathrm{T}_{1} \text { planar }}^{z}\right) \\
\mathbf{B}_{2}= & \frac{1}{2}\left(2 m_{\mathrm{T}_{1} \text { planar }}^{x},-m_{\mathrm{T}_{2}}^{y}+\sqrt{3} m_{\mathrm{T}_{1} \text { planar }}^{y},-m_{\mathrm{T}_{2}}^{z}\right. \\
& \left.-\sqrt{3} m_{\mathrm{T}_{1} \text { planar }}^{z}\right)
\end{aligned}
$$

which each separately obey their own Gauss's law:

$$
\nabla \cdot \mathbf{B}_{1}=0, \quad \nabla \cdot \mathbf{B}_{2}=0 .
$$

We can therefore write

$$
\mathbf{B}_{1}=\nabla \times \mathbf{A}_{1}, \quad \mathbf{B}_{2}=\nabla \times \mathbf{A}_{2},
$$

and the theory has two independent $\mathrm{U}(1)$ gauge degrees of freedom.

The free energy associated with the fluctuations of these fields is of entropic origin [65]. The only choice of Gaussian free-energy consistent with both the point-group symmetry and the $U(1)$ symmetry of $\mathcal{H}_{\mathrm{XXZ}}$ is

$$
\begin{aligned}
\mathcal{F}_{\mathrm{SL}_{\perp}} & =\frac{T}{V} \int d^{3} r \lambda\left(\mathbf{B}_{1}^{2}+\mathbf{B}_{2}^{2}\right) \\
& =\frac{T}{V} \int d^{3} r \lambda\left[\left(\nabla \times \mathbf{A}_{1}\right)^{2}+\left(\nabla \times \mathbf{A}_{2}\right)^{2}\right],
\end{aligned}
$$

where the coefficient $\lambda$ can be determined through fits to simulation or a large-N expansion $[55,72]$.

It follows from the existence of the conserved fluxes $\mathbf{B}_{1}$ and $\mathbf{B}_{2}$ and the free-energy Eq. (11) that $\mathrm{SL}_{\perp}$ is a Coulomb phase with algebraic correlations [65]. The validity of this description is demonstrated in Fig. 4, where we compare analytic calculations of the flux structure factor

$$
S_{\mathbf{B}_{\mu}}^{\alpha \beta}(\mathbf{q})=\left\langle B_{\mu}^{\alpha}(-\mathbf{q}) B_{\mu}^{\beta}(\mathbf{q})\right\rangle,
$$

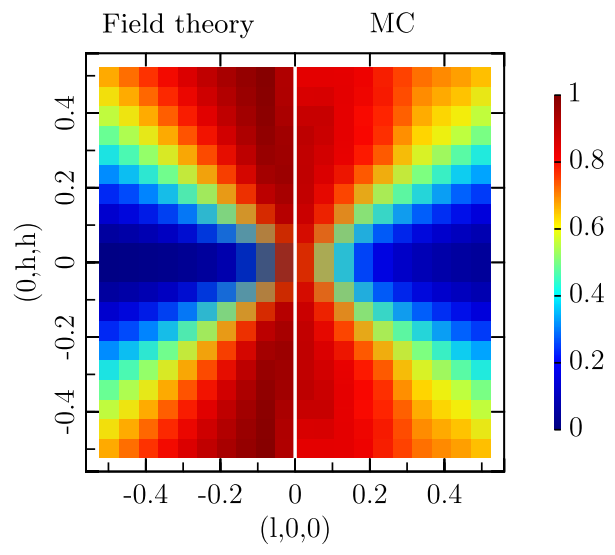

FIG. 4. $\mathrm{U}(1) \times \mathrm{U}(1)$ gauge structure of the easy-plane spin liquid $\left(\mathrm{SL}_{\perp}\right)$, as demonstrated by pinch points in equal-time structure factors $S_{\mathbf{B}_{\mu}}^{\alpha \beta}(\mathbf{q})$ [Eq. (12)]. Left half of panel: Structure factor $S_{\mathbf{B}_{1}}^{x x}(\mathbf{q})$ as calculated from the theory Eq. (11), with $\lambda=1$. Right half of panel: Structure factor $S_{\mathbf{B}_{1}}^{x x}(\mathbf{q})$ as calculated in classical Monte Carlo simulations of $\mathcal{H}_{\text {XXZ }}$ [Eq. (1)]. The pinch point centered on $\mathbf{q}=(0,0,0)$ follows from the zero-divergence conditions on the fields $\mathbf{B}_{\mu}$ [Eq. (9)]. Simulations were carried out for a cubic cluster of $N=8192$ spins, with $J^{ \pm} / J_{z z}=-1$, $T=0.01 J_{\mathrm{zz}}$, as described in Appendix E.

based on Eq. (11) with the results of Monte Carlo simulation. Pinch-point singularities are clearly seen in both analytic and numerical calculations. It is the same fluctuations of $\mathbf{B}_{1}$ and $\mathbf{B}_{2}$ that are responsible for the characteristic pinch-point structures in the (spin) structure factor measured by neutron scattering, as shown in Fig. 2(b).

At finite temperature, we anticipate that the spin liquid $\mathrm{SL}_{\perp}$ will be perturbatively stable against terms such as $\delta \mathcal{H}_{ \pm \pm}$[Eq. (6)], which retain the point-group symmetry of the lattice but lift the $\mathrm{U}(1)$ symmetry of the spins. In this case, the free energy will be modified:

$$
\begin{aligned}
\mathcal{F}_{\mathrm{SL}_{\perp}} & \rightarrow \mathcal{F}_{\mathrm{SL}_{\perp}}+\delta \mathcal{F}_{\mathrm{SL}_{\perp}} \\
\delta \mathcal{F}_{\mathrm{SL}_{\perp}} & =\frac{T}{V} \int d^{3} r \lambda^{\prime}\left\{\left(B_{1}^{x}\right)^{2}-\frac{1}{2}\left[\left(B_{1}^{y}\right)^{2}+\left(B_{1}^{z}\right)^{2}\right]\right. \\
& \left.-\left(B_{2}^{x}\right)^{2}+\frac{1}{2}\left[\left(B_{2}^{y}\right)^{2}+\left(B_{2}^{z}\right)^{2}\right]-\sqrt{3}\left[B_{1}^{z} B_{2}^{z}-B_{1}^{y} B_{2}^{y}\right]\right\}
\end{aligned}
$$

This form of free energy will still lead to pinch points in $S_{\mathbf{B}_{\mu}}^{\alpha \beta}(\mathbf{q})$ and $S(\mathbf{q})$, but these will take on a more anisotropic character.

\section{DYNAMICS IN THE SPIN-NEMATIC PHASE}

For temperatures $T<T_{\mathrm{SN}}$, the easy-plane spin liquid $\left(\mathrm{SL}_{\perp}\right)$ gives way to a phase with hidden spin-nematic order, 
labeled $\mathrm{SN}_{\perp}$ in Fig. 1. As far as the dipole moments of spins are concerned, the spin-nematic phase is disordered, and neutron scattering experiments would reveal algebraic correlations, as in the spin liquid $\mathrm{SL}_{\perp}$. However, the pinch points in $S(\mathbf{q})$ [cf. Fig. 2(b)] hide a great wealth of interesting spin excitations.

To better understand the dynamics of the spin-nematic phase, we have calculated the dynamical structure factor $S(\mathbf{q}, \omega)$, within a semiclassical molecular-dynamics (MD) simulation, using the methods described in Ref. [77]. Relevant definitions are given in Appendix C. For $\omega / J_{\mathrm{zz}} \lesssim 0.2, S(\mathbf{q}, \omega)$ presents a featureless, nondispersing continuum [Fig. 5(a)]. Relics of dispersing excitations are visible in $S(\mathbf{q}, \omega)$ at higher energies, but these are explicitly not Goldstone modes and have nothing to do with the hidden spin-nematic order. Examining the evolution of $S(\mathbf{q}, \omega)$ as a function of temperature, we find that results for $S(\mathbf{q}, \omega)$ in the spin-nematic phase for $T<T_{\mathrm{SN}}$ are very similar to those found in the spin liquid $\mathrm{SL}_{\perp}$ for $T>T_{\mathrm{SN}}$.

Incoherent, nondispersing structure of the type shown in Fig. 5(a) is reminiscent of theoretical predictions [78-80] and experimental measurements $[43,81,82]$, for a wide range of different spin liquids. In a quantum spin liquid, the presence of a nondispersing continuum reflects the fact that, unlike conventional spin waves (magnons), single elementary excitations of a spin liquid cannot be created by local processes. It follows that, when a neutron scatters from a spin liquid, the energy, momentum, and angular momentum (spin) transferred are not absorbed by a single excitation with a well-defined energy and momentum, but rather they are shared between multiple excitations [83]. In the semiclassical limit studied here, it is probably unsafe to attribute such a continuum to fractionalized excitations [62]. However, the fact that $S(\mathbf{q}, \omega)$ only records dipolar spin correlations obscures a more important fact - the spin-nematic order that is present for $T<T_{\mathrm{SN}}$ that breaks a continuous, $\mathrm{U}(1)$ symmetry of the Hamiltonian. By Goldstone's theorem, it must, therefore, also support gapless Goldstone modes.

In order to resolve this conundrum, it is necessary to examine the dynamical correlations of the quadrupole moments of spin. In Fig. 5(b), we present MD simulation results for the dynamical susceptibility $\chi_{\mathrm{Q}^{\text {site }}}(\mathbf{q}, \omega)$, which measures fluctuations of the on-site quadrupolar moments, which are well defined for classical spins [cf. Appendix B]. A sharp excitation, with dispersion

$$
\omega \approx v_{Q}\left|\mathbf{q}-\mathbf{q}_{\mathbf{r l}}\right|
$$

can now be resolved close to the zone centers with $\mathbf{q}_{\mathrm{rl}}=(0,0,0),(1,1,1),(2,2,2)$. These are the same zone centers for which the Bragg peaks associated with the hidden spin-nematic order $\mathrm{SN}_{\perp}$ would occur in a quadrupolar structure factor, which might, in principle, be measured in resonant $\mathrm{x}$-ray experiments [84].

At present, relatively little is known about the dynamical properties of spin-nematic states. Field-theoretic analysis [70,85-87], based on the symmetry of the order parameter, predicts that spin-nematics support gapless Goldstone

(a) Dynamical structure factor for spins, $S(\mathbf{q}, \omega)$

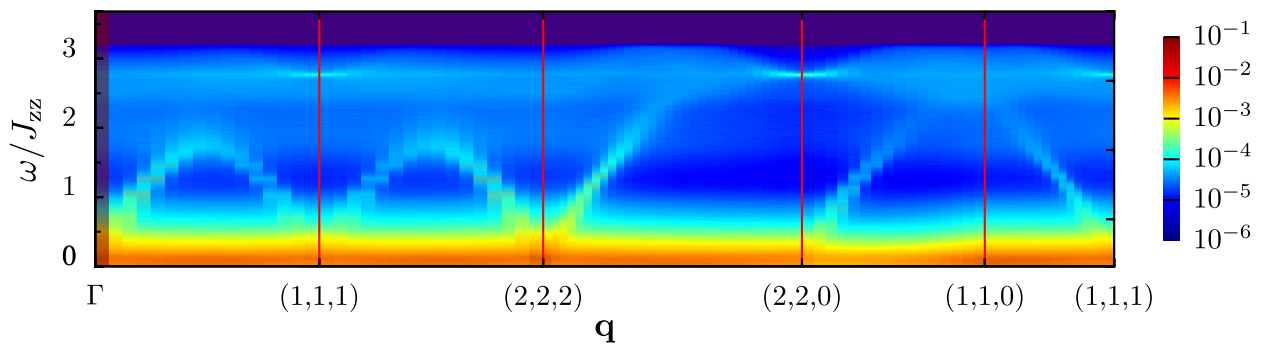

(b) Dynamical susceptibility for quadrupoles, $\chi_{Q_{\perp}^{\text {site }}}(\mathbf{q}, \omega)$

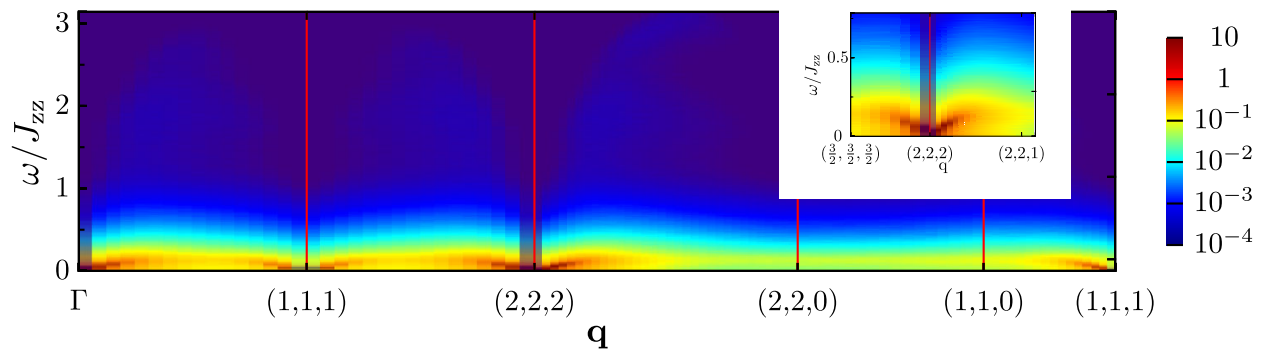

FIG. 5. Spin dynamics in the phase with hidden spin-nematic order $\left(\mathrm{SN}_{\perp}\right)$. (a) Dynamical structure factor for spin degrees of freedom, $S(\mathbf{q}, \omega)$, showing gapless continuum of excitations at low energies. (b) Dynamical susceptibility for fluctuations of on-site quadrupole moments, $\chi_{Q^{\text {site }}}(\mathbf{q}, \omega)$ [cf. Appendix F]. Inset: Details of correlations at low energies near the zone center $\mathbf{q}_{\mathrm{rl}}=(2,2,2)$, showing a linearly dispersing Goldstone mode at low energies. Results are taken from molecular-dynamics simulations of $\mathcal{H}_{\text {xxz }}$ [Eq. (1)] for a cluster of $N=65536$ spins, with $J_{ \pm} / J_{\mathrm{zz}}=-1.0, T / J_{\mathrm{zz}}=0.002$. 
modes, visible in $\chi_{Q_{\perp}}(\mathbf{q}, \omega)$. This Goldstone mode has dispersion $\omega \propto|\mathbf{q}|$ [cf. Eq. (15)], and at zero temperature the associated intensity diverges as $\sim 1 / \omega$ for $\omega \rightarrow 0$ [70]. The same behavior is seen in "flavor-wave" calculations and QMC simulations of spin-1 models constructed to support quadrupolar order [88-90]. The dynamics of the spin-1/2 frustrated ferromagnetic spin chain have also been studied using density matrix renormalization group (DMRG) calculations, and they reveal a broad continuum of excitations at high energies [91]. However, because of the absence of long-range order, no Goldstone modes can be resolved. A continuum of excitations at high energies is also found in calculations for two-dimensional frustrated ferromagnets, within a slave-particle mean-field picture [92].

Our MD simulations of $\mathrm{SN}_{\perp}$ clearly reveal a linearly dispersing Goldstone mode, with intensity that diverges for $\omega \rightarrow 0$ [cf. inset in Fig. 5(b)]. The form of this divergence is $\sim 1 / \omega^{2}$, rather than $\sim 1 / \omega$. This follows from the fact that simulations are carried out at finite temperature and probe thermal, rather than quantum, fluctuations. Most striking, however, is the broad continuum of excitations visible in both spin and quadrupole structure factors. It is also interesting to note that the way in which the Goldstone mode "dissolves" into this continuum bears some resemblance to what is seen in QMC simulations of a spin-1 model at higher temperature [90]. Overall, the picture that emerges from MD simulation is consistent with all known facts about spin-nematics and should provide a reliable guide for comparison with experiment.

Further details of the spin dynamics in the spin-nematic phase and specifically the characterization of the Goldstone mode are given in Appendix F. We note that the U(1) symmetry of $\mathcal{H}_{\mathrm{XXZ}}$ [Eq. (1)] is not a necessary condition for spin-nematic order to exist. However, if this symmetry were broken, the low-energy (pseudo-)Goldstone mode associated with $\mathrm{SN}_{\perp}$ would acquire a small gap.

\section{DISCUSSION}

Spin liquids [1-3] and spin nematics [66-68] are prime examples of unconventional states of matter and have many unusual and interesting properties. The experimental search for these exotic states has a long history, with many twists and turns, and not a few dead ends. Given this, finding both in one simple, canonical, and experimentally motivated model is remarkable. It is therefore worth considering the possibilities for observing the unconventional spin liquid $\mathrm{SL}_{\perp}$ and the spin nematic $\mathrm{SN}_{\perp}$ in the type of rare-earth pyrochlore magnet described by Eq. (1).

In the case of $\mathrm{SN}_{\perp}$, it is important to make a distinction between the type of spin-nematic order considered in this manuscript, which is driven by fluctuations, and the quadrupolar or octupolar order, which can arise directly from the ground states of rare-earth ions. Here we particularly have in mind the non-Kramers ions $\operatorname{Pr}^{3+}[9,18,43]$ and $\mathrm{Tb}^{3+}[9,38,44,93]$ and Kramers doublets of dipolar- octupolar character, such as $\mathrm{Nd}^{3+}$ [51] and $\mathrm{Ce}^{3+}$ [94]. For these ions, quadrupolar or octupolar order may occur as a "classical" order of the transverse part of the pseudospins $\mathrm{S}_{i}$. The multipolar character of the order follows from the symmetry of the crystal-field ground state (doublet) of the magnetic ion, which is described by $\mathrm{S}_{i}$. Where multipolar order of this kind occurs, experiments that probe the dynamics of dipoles will see a gapped response and a sharp excitation spectrum. In contrast, in the easy-plane spin nematic $\mathrm{SN}_{\perp}$, dipole moments remain in a spin-liquidlike state, with strong fluctuations at low temperature and a broad, gapless response coexisting with the hidden nematic order [Fig. 5(a)].

Where, then, might we observe these unusual magnetic states? Further experimental work will be necessary to definitively answer this question, but there are already a few trails to follow. In particular, the Pr-based pyrochlores have the recommended single-ion and interaction anisotropies $[9,18,43]$. Coupling parameters of $\mathrm{Pr}_{2} \mathrm{Zr}_{2} \mathrm{O}_{7}$, for example, have been suggested to sit in the $\mathrm{AF}_{\perp}$ phase of Fig. 1 [43], although it seems that the coupling of structural disorder to the non-Kramers doublets plays a significant role $[23,33,95,96]$. Since chemical pressure has already proven to be a useful tool to move a family of compounds across a phase diagram [41,49,75,97,98], $\quad \operatorname{Pr}_{2} \mathrm{X}_{2} \mathrm{O}_{7}$ $(\mathrm{X}=\mathrm{Sn}, \mathrm{Hf}, \mathrm{Pb})$ are promising candidates to investigate, with ferromagnetic correlations consistent with positive $J_{\mathrm{zz}}$ and no dipole order yet observed [26,31,32,40,99].

The notion of hidden order also resonates with the elusive physics of $\mathrm{Yb}$-based pyrochlores. As far as we know, $\mathrm{Yb}$ pyrochlores lie in a different regime of magnetic interactions than the $\mathcal{H}_{\mathrm{XXZ}}$ model of Eq. (1). Specifically, experiments on $\mathrm{Yb}_{2} \mathrm{Ti}_{2} \mathrm{O}_{7}$ point to an unfrustrated value of $J_{ \pm}>0[11,100,101]$ and to an important role for other competing exchange interactions. In light of this, the properties of that particular material seem to be connected with a different phase boundary from that associated with $\mathrm{SL}_{\perp}[45,49,75]$. That being said, some of the similarities between our results and the $\mathrm{Yb}$ pyrochlores are striking: a gapless continuum of spin excitations, oblivious to thermodynamic phase transitions [41,102-104] [Fig. 5(a)] and robust in temperature up to a broad feature in specific heat [41] (in the present article, between $\mathrm{pHAF}$ and $\mathrm{SL}_{\perp}$ ). While the magnetic order in $\mathrm{Yb}$-pyrochlores is, at least partially, an order of dipolar moments [27,36,37,105-107], recent experiments have indicated that the primary order parameter may be "hidden," and distinct from a standard dipole order [41]. Thus, while the specific case developed in this article probably does not apply to the $\mathrm{Yb}$ pyrochlores, related physics may be at play.

Furthermore, since the spin-nematic phase $\mathrm{SN}_{\perp}$ is found within the spin liquid $\mathrm{SL}_{\perp}$ (Fig. 1), this work provides a prototype for the peaceful coexistence of emergent gauge fields and long-range order. In this sense, $\mathrm{SN}_{\perp}$ is an interesting new addition to the other phases where gauge 
fluctuations and broken symmetries coexist, such as the Coulombic ferromagnet $[17,108]$ and states with magneticmoment fragmentation [109], as recently observed in $\mathrm{Nd}_{2} \mathrm{Zr}_{2} \mathrm{O}_{7}$ [42,110] and $\mathrm{Ho}_{2} \mathrm{Ir}_{2} \mathrm{O}_{7}$ [111].

We also note that many other magnetic systems outside the rare-earth oxides $\mathrm{R}_{2} \mathrm{X}_{2} \mathrm{O}_{7}$ feature moments located on a pyrochlore lattice. Of particular interest are materials such as $\mathrm{NaCaCo}_{2} \mathrm{~F}_{7}$ and $\mathrm{NaSrCo}_{2} \mathrm{~F}_{7}[112,113]$ that boast $\mathrm{XY}$-like interactions with much higher energy scales than those observed in the rare-earth oxides. If such a case could be found with frustrated transverse coupling $J_{ \pm}<0$, then it would render the physics discussed here accessible at a much more amenable temperature range.

In almost all spin-liquid candidates, the role of quenched structural and chemical disorder is an important issue [33,38,95,96,114-119]. Depending on the type and strength of disorder, its consequences can vary. It is worth noting, however, that disorder is not necessarily deleterious to spin-liquid physics. It is known, for example, that weak disorder in non-Kramers pyrochlores, which leads to splittings in the low-energy non-Kramers doublet, can actually play a role in promoting a $\mathrm{U}(1)$ spin-liquid ground state [23]. The spin-liquid states discussed in this manuscript do not depend on the translational symmetry of the Hamiltonian, but rather on the emergent gauge symmetries that arise from the local constraints in the ground state [Eq. (5)]. Thus, as long as the disorder is not so strong that these constraints are strongly violated, the essence of the spin liquids should be maintained in the presence of disorder, at least at finite temperature. For sufficiently strong disorder or sufficiently low temperature, disorder may lead to order by disorder or glassiness [120,121]. A quantitative study of the effects of disorder on the phase diagram in Fig. 1 is a large undertaking and is beyond the scope of the present work but may be an interesting direction for future consideration.

\section{SUMMARY AND CONCLUSIONS}

"Quantum spin ice," in which magnetic ions on a pyrochlore lattice interact through highly anisotropic exchange interactions, has become an important paradigm in the search for quantum spin liquids. In this article, we use large-scale classical Monte Carlo simulation to explore the physics of the canonical model of a quantum spin ice, the XXZ model on a pyrochlore lattice $\mathcal{H}_{\text {XXZ }}$ [Eq. (1)]. We find that this model has far more to offer than spin ice alone, supporting three distinct types of spin liquid, each with a different emergent gauge symmetry. Each of these spin liquids has a different signature in neutron scattering (Fig. 2). The states found include a completely new form of spin liquid, described by a $\mathrm{U}(1) \times \mathrm{U}(1)$ gauge theory. At low temperatures, this novel spin liquid undergoes a phase transition to a state with hidden spin-nematic order (Fig. 1) but retains algebraic correlations of the spin dipoles. We have studied the excitations of this phase using state-of-the- art dynamical simulations, revealing a sharply defined Goldstone mode that would be hidden from conventional neutron scattering techniques.

So far as experiment is concerned, the main lesson of these results is that "quantum spin-ice" materials can play host to a great many different spin-liquid and (hidden-) order phases, even where they are described by a Hamiltonian as simple as $\mathcal{H}_{\mathrm{XXZ}}$ [Eq. (1)]. This reinforces the point that pinch points in pyrochlore magnets need not imply spin ice [65,72]. The existence of a sharp Goldstone mode in the nematic phase $\mathrm{SN}_{\perp}$ also serves as a salutary reminder that broad, nondispersing continua of excitations can hide a multitude of secrets (Fig. 5).

From the theoretical point of view, this work identifies a new spin liquid, a novel spin-nematic phase, and opens an interesting new perspective on the way in which different spin liquids can compete. The effect of quantum fluctuations on the phase diagram shown in Fig. 1 for $J_{ \pm}<0$ remains a subject for future study. However, experience with QMC simulation of $\mathcal{H}_{\mathrm{XXZ}}$ [Eq. (1)] for $J_{ \pm}>0$ suggests that quantitative values of the crossover temperature $T_{2}^{*}$ and $T_{3}^{*}$ may be substantially renormalized, but that the qualitative structure of the phase diagram should remain the same down to very low temperatures $[14,21,25]$. The high-symmetry point, $J_{ \pm} / J_{\mathrm{zz}}=-1 / 2$, is also a highsymmetry point for quantum spins, and so remains the anchor for the spin liquid pHAF. Nonetheless, the fate of this $\mathrm{U}(1) \times \mathrm{U}(1) \times \mathrm{U}(1)$ spin liquid for quantum spins at $T=0$ remains an open question $[57,58,60,122]$. To the best of our knowledge, quantum analogues of the new spin liquid, $\mathrm{SL}_{\perp}$, which has a $\mathrm{U}(1) \times \mathrm{U}(1)$ gauge structure, remain unexplored [123]. However, it seems reasonable to speculate that quantum effects will enhance, rather than suppress, the fluctuations that drive $\mathrm{SL}_{\perp}$ and $\mathrm{pHAF}$, and that the phase $\mathrm{SN}_{\perp}$ will survive as hidden quantum spinnematic order, within a quantum spin liquid. Preliminary numerical results for the spin-1/2 model at high temperatures are entirely consistent with the topology of the phase diagram shown in Fig. 1 [124]. All of these questions open exciting avenues for future research.

\section{ACKNOWLEDGMENTS}

We are pleased to acknowledge many helpful discussions with Karim Essafi, Jaan Oitmaa, and Rajiv Singh. This work was supported by the Theory of Quantum Matter Unit of the Okinawa Institute of Science and Technology Graduate University (OIST). Numerical calculations were carried out using HPC facilities provided by OIST.

\section{APPENDIX A: DEFINITION OF LOCAL-COORDINATE FRAME}

We describe the local-coordinate frame that is defined for four spins on a pyrochlore tetrahedron $S_{0}, S_{1}, S_{2}, S_{3}$, occupying positions 


$$
\begin{aligned}
& \mathbf{r}_{0}=\frac{a}{8}(1,1,1) \quad \mathbf{r}_{1}=\frac{a}{8}(1,-1,-1) \\
& \mathbf{r}_{2}=\frac{a}{8}(-1,1,-1) \quad \mathbf{r}_{3}=\frac{a}{8}(-1,-1,1)
\end{aligned}
$$

where $a$ is the length of a cubic, 16-site unit cell of the pyrochlore lattice.

The pseudospins in the global, crystal, coordinate frame $\mathbf{S}_{i}$ relate to the pseudospins in the local frame $S_{i}$ [Eq. (1)] as

$$
\mathbf{S}_{i}=\mathbf{x}_{i}^{\text {local }} \mathbf{S}_{i}^{x}+\mathbf{y}_{i}^{\text {local }} \mathbf{S}_{i}^{y}+\mathbf{z}_{i}^{\text {local }} \mathbf{S}_{i}^{z},
$$

where

$$
\begin{array}{rlrl}
\mathbf{z}_{0}^{\text {local }} & =\frac{1}{\sqrt{3}}(1,1,1) & \mathbf{z}_{1}^{\text {local }}=\frac{1}{\sqrt{3}}(1,-1,-1) \\
\mathbf{z}_{2}^{\text {local }} & =\frac{1}{\sqrt{3}}(-1,1,-1) & \mathbf{z}_{3}^{\text {local }} & =\frac{1}{\sqrt{3}}(-1,-1,1), \\
\mathbf{x}_{0}^{\text {local }} & =\frac{1}{\sqrt{6}}(-2,1,1) & \mathbf{x}_{1}^{\text {local }} & =\frac{1}{\sqrt{6}}(-2,-1,-1) \\
\mathbf{x}_{2}^{\text {local }} & =\frac{1}{\sqrt{6}}(2,1,-1) & \mathbf{x}_{3}^{\text {local }} & =\frac{1}{\sqrt{6}}(2,-1,1),
\end{array}
$$

and

$$
\begin{array}{ll}
\mathbf{y}_{0}^{\text {local }}=\frac{1}{\sqrt{2}}(0,-1,1) & \mathbf{y}_{1}^{\text {local }}=\frac{1}{\sqrt{2}}(0,1,-1) \\
\mathbf{y}_{2}^{\text {local }}=\frac{1}{\sqrt{2}}(0,-1,-1) & \mathbf{y}_{3}^{\text {local }}=\frac{1}{\sqrt{2}}(0,1,1) .
\end{array}
$$

We have used this relationship between the local coordinate frame of Eq. (1) and the crystal coordinate frame to plot a representation of the bond quadrupolar order in real space in Fig. 1(b). The ellipsoid on each bond $i j$ in Fig. 1(b) has principal axes aligned along the cubic axes of the pyrochlore lattice, with the length of each principal axis given by

$$
l_{\alpha}=4\left(c+\left\langle S_{i}^{\alpha} S_{j}^{\alpha}\right\rangle\right)
$$

where $c=0.08$ is chosen to make the figure readable and $S_{i}^{\alpha}$ is the component of spin $i$ in the global frame, along crystal axis $\alpha=x, y, z$.

\section{APPENDIX B: DETAILS OF THE NUMERICAL DETERMINATION OF THE PHASE DIAGRAM}

The phase diagram shown in Fig. 1 is extracted from classical Monte Carlo (MC) simulations of the quantum spin-ice model, $\mathcal{H}_{\mathrm{XXZ}}$ [Eq. (1)]. Spins are treated as classical vectors with fixed length $\left|S_{i}\right|=\frac{1}{2}$. These simulations are carried out for a cubic cluster of 8192 spins, using a single spin-flip algorithm combined with simulated annealing, parallel tempering, and overrelaxation. The phase diagram is obtained using 75000 simulated annealing steps using a stepwise decrease of temperature, starting from $T=10 J_{\mathrm{zz}}$ down to the target temperature. Each annealing step consists of ten Monte Carlo steps (a Monte Carlo step consists of a full sweep of the lattice combined with overrelaxation). The simulated annealing is followed by 1000 parallel tempering steps with 500 Monte Carlo steps in between and then by 200000 Monte Carlo steps for thermalization at fixed temperature. Measurements consist of 200000 samples separated by ten Monte Carlo steps and combined to parallel tempering every 50 measures. We use 256 different replicas with temperature set in linear scale for $J_{ \pm} / J_{\mathrm{zz}}>-\frac{1}{2}$ and 256 temperatures in logarithmic scale for $J_{ \pm} / J_{\mathrm{zz}} \leq-\frac{1}{2}$.

The phase boundary of the antiferromagnetically ordered $\left(\mathrm{AF}_{\perp}\right)$ phase, $T_{N}$, is extracted from the susceptibility of the relevant order parameter, $\mathbf{m}_{\mathrm{E}}$, as defined in Appendix D.

The crossover scale for the spin-ice regime (SI), $T_{1}^{*}$, is estimated from the Schottky-like peak in the heat capacity.

The crossover scale $T_{3}^{*}$ for the spin liquid pHAF is estimated from the Curie-law crossover shown in Fig. 6.

For $J_{ \pm}<-\frac{1}{2}$, the crossover scale $T_{2}^{*}$ is associated with a weakening of the correlations of the local $z$-components of the spins. This can be observed by measuring the susceptibility, $\chi_{\mathrm{T}_{1} \text { ।ce }}(T)$, of the field $\mathbf{m}_{\mathrm{T}_{1} \text { Ice }}$, defined in Appendix D. Decreasing the temperature for $-1<\left(J_{ \pm} / J_{z z}\right)<-0.5$, the quantity $T \chi_{\mathrm{T}_{1} \text { lce }}(T)$ first increases during the crossover from the paramagnet to $\mathrm{pHAF}$ and then drops as the system enters $\mathrm{SL}_{\perp}$. We define the crossover temperature $T_{2}^{*}$ as the point at which the quantity $T \chi_{T_{1} \text { ice }}(T)$ drops below its infinite temperature value

$$
T_{2}^{*} \chi_{\mathrm{T}_{1} \text { ice }}\left(T_{2}^{*}\right)=\lim _{T \rightarrow \infty} T \chi_{\mathrm{T}_{1} \text { ice }}(T)
$$

This is illustrated in Fig. 7(b).

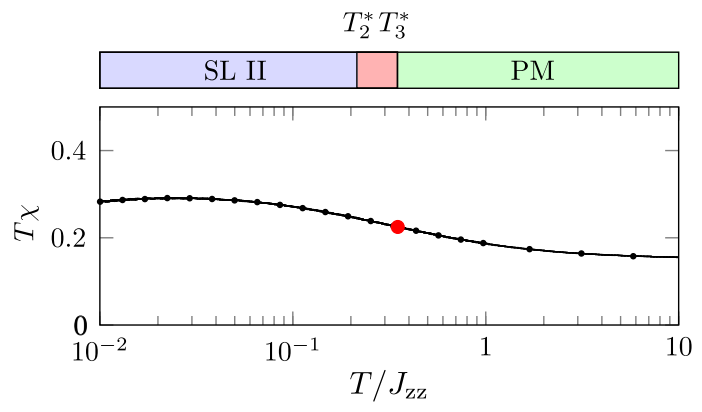

FIG. 6. Crossover in the magnetic susceptibility $\chi(T)$, from a traditional Curie law in the high-temperature paramagnet to a low-temperature Curie law in the spin liquids, as seen by different plateaus in the function $T \chi$, plotted as a function of $\log (T)$. The crossover temperature $T_{3}^{*} / J_{\mathrm{zz}} \approx 0.3$ (red dot) is estimated from the point of inflection of $T \chi$. The extraction of the crossover temperature $T_{2}^{*}$ is explained in Fig. 7. Results are taken from classical Monte Carlo simulations of $\mathcal{H}_{\mathrm{XXZ}}$ [Eq. (1)], for a cubic cluster of $N=8192$ spins, with $J_{ \pm} / J_{\mathrm{zz}}=-1$. 
(a) Specific heat

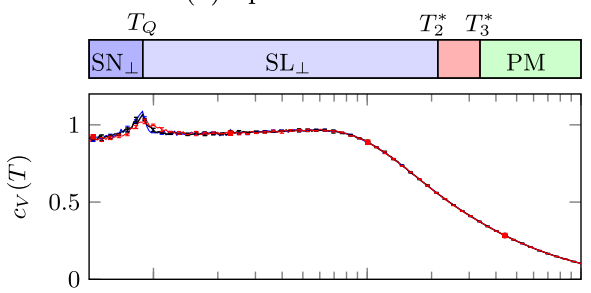

(b) $T \chi_{\mathrm{T}_{1} \text { ice }}(T)$

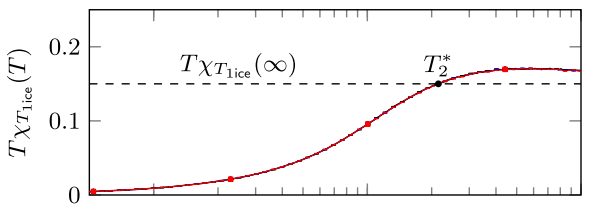

(c) Spin-nematic bond order parameter

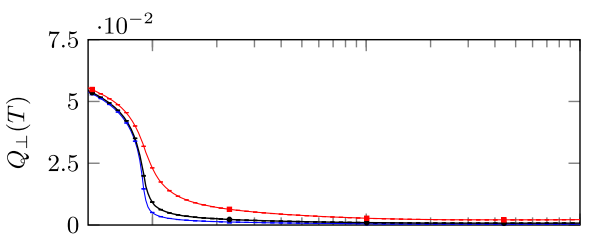

(d) Spin-nematic site order parameter

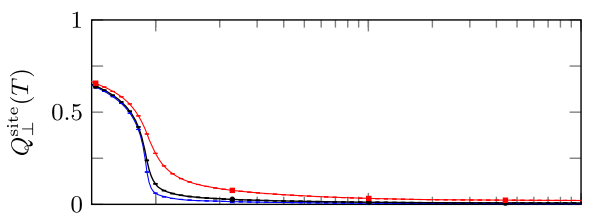

(e) Order-parameter susceptibility

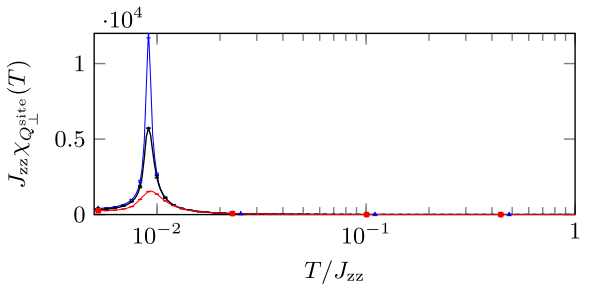

FIG. 7. Thermodynamics of the QSI in the region of spin-nematic order. (a) Specific heat $c_{V}(T)$, showing an upturn followed by a shallow maximum in the region of the crossover into the spin liquid $\mathrm{SL}_{\perp}$ at $T_{2}^{*} / J_{\mathrm{zz}} \sim 10^{-1}$, and small peak associated with the onset of spin-nematic order at $T_{\mathrm{SN}} / J_{\mathrm{zz}} \approx 10^{-2}$. (b) Correlation function $T \chi_{\mathrm{T}_{1} \text { ice }}(T)$ used to determine the crossover temperature into the spin liquid $\mathrm{SL}_{\perp} \cdot \chi_{\mathrm{T}_{1} \text { ice }}$ is the susceptibility of the field $\mathbf{m}_{\mathrm{T}_{1} \text { ice }}$ defined in Appendix D. The crossover temperature $T_{2}^{*}$ is defined as the point at which $T \chi_{\mathrm{T}_{1} \text { ice }}$ drops below its infinite temperature limit [Eq. (B1)]. (c) Norm of the bond order parameter $\mathbf{Q}_{\perp}(T)$ [cf. Eq. (B2)], showing a continuous phase transition into the phase with hidden spin-nematic order at $T_{\mathrm{SN}} / J_{\mathrm{zz}} \approx 10^{-2}$. (d) Norm of the site order parameter $\mathbf{Q}_{\perp}^{\text {site }}(T)$ [cf. Eq. (B2)], also showing the phase transition into the spin-nematic phase. (e) Order-parameter susceptibility $\chi_{\mathrm{Q}_{\perp}^{\text {site }}}(T)$ [Eq. (B3)], used to estimate the transition temperature $T_{\mathrm{SN}}$. Results are taken from classical Monte Carlo simulation of $\mathcal{H}_{\mathrm{XXZ}}$ [Eq. (1)], for cubic clusters of $N=1024,8192$, and 27648 spins, with $J_{ \pm} / J_{\mathrm{zz}}=-1$.
For quantum, spin-1/2 moments, the onset of spinnematic order is heralded by the bond-based order parameter Eq. (2). However, for the purpose of classical simulation, it is sufficient to consider the simpler, site-based order parameter

$$
\mathbf{Q}_{\perp}^{\text {site }}=\frac{4}{N} \sum_{i}\left(\begin{array}{c}
\mathrm{S}_{i}^{x 2}-\mathrm{S}_{i}^{y 2} \\
2 \mathrm{~S}_{i}^{x} \mathrm{~S}_{i}^{y}
\end{array}\right) .
$$

Please note the prefactor of 4 in the definition of the spin-nematic site order parameter [Eq. (B2)] to account for the spin length $\left|S_{i}\right|=\frac{1}{2}$. The onset of spin-nematic order in simulations can be observed in either the site-based [cf. Fig. 7(d)] or bond-based order parameters [cf. Fig. 7(c)]. However, for simplicity, the values of the spin-nematic ordering temperature $T_{\mathrm{SN}}$ shown in Fig. 1 are extracted from the peak in the order-parameter susceptibility,

$$
\chi_{Q_{\perp}^{\text {site }}}=\frac{N}{T}\left(\left\langle\mathbf{Q}_{\perp}^{\text {site2 }}\right\rangle-\left\langle\left|\mathbf{Q}_{\perp}^{\text {site }}\right|\right\rangle^{2}\right),
$$

associated with the site-based order parameter, Eq. (B2) [cf. Fig. 7(e)].

Figure 7 is obtained using 300 temperatures in logarithmic scale covering three orders of magnitude, parallel tempering every 100 Monte Carlo steps, simulated annealing, and thermalization at temperature $T$ for 100000 Monte Carlo steps each. Measurements consist of 100000 different samples with ten Monte Carlo steps between each sample. Error bars were estimated by comparing the results of three independent runs of the simulation.

\section{APPENDIX C: DEFINITIONS OF DYNAMICAL STRUCTURE FACTORS}

In Fig. 2 we show predictions for neutron scattering experiments, based on the equal-time (i.e., energy-integrated) structure factor

$$
S(\mathbf{q})=\int d \omega S(\mathbf{q}, \omega)
$$

where the dynamical structure factor $S(\mathbf{q}, \omega)$ is defined through

$$
\begin{aligned}
S(\mathbf{q}, \omega) & =\sum_{\alpha \beta}\left(\delta_{\alpha \beta}-\frac{q_{\alpha} q_{\beta}}{q^{2}}\right)\left\langle m^{\alpha}(-\mathbf{q}, \omega) m^{\beta}(\mathbf{q}, \omega)\right\rangle \\
m^{\alpha}(\mathbf{q}, \omega) & =\sum_{i \beta \gamma} R_{i}^{\alpha \beta} g_{i}^{\beta \gamma}\left(\int \mathrm{S}_{i}^{\gamma}(t) e^{i \omega t} d t\right) e^{i \mathbf{q} \cdot \mathbf{r}_{i}}
\end{aligned}
$$

and the $g_{i}^{\beta \gamma}$ is the $g$-tensor written in the local coordinate frame [49]. For simplicity, we have here taken $g_{i}^{\beta \gamma}=2 \delta_{\beta \gamma}$ for all of the calculations in this paper. $R_{i}^{\alpha \beta}$ is a rotation matrix that rotates from the local coordinate frame on site $i$, to the global, crystal coordinate frame. The definition of the 
local coordinate frame is given in Appendix A. Results for $S(\mathbf{q})$ are shown in the left-half panels of Fig. 2. These results are taken from classical MC simulations of $\mathcal{H}_{\mathrm{XXz}}$ at a given temperature, with further averaging provided by numerically integrating the semiclassical equations of motion for the spins. This secondary MD simulation is carried out using methods described in Ref. [77].

It is also useful to decompose the structure factor into the spin-flip (SF) and non-spin-flip (NSF) channels measured in polarized neutron-scattering experiments:

$$
\begin{aligned}
S_{\mathrm{SF}}(\mathbf{q}) & =\frac{1}{q^{2}} \int d \omega\left\langle|\mathbf{m}(\mathbf{q}, \omega) \cdot(\hat{\mathbf{n}} \times \mathbf{q})|^{2}\right\rangle \\
S_{\mathrm{NSF}}(\mathbf{q}) & =\int d \omega\left\langle|\mathbf{m}(\mathbf{q}, \omega) \cdot \hat{\mathbf{n}}|^{2}\right\rangle,
\end{aligned}
$$

where $\hat{\mathbf{n}}$ is the direction of polarization of the neutron magnetic moment. Following Fennell et al. [46], we take $\hat{\mathbf{n}}=(1,-1,0) / \sqrt{2}$. Simulation results for $S_{\mathrm{SF}}(\mathbf{q})$ and $S_{\mathrm{NSF}}(\mathbf{q})$ are shown in the right-half panels of Fig. 2.

We also use MD simulation to calculate the dynamical structure factor $S(\mathbf{q}, \omega)$. Results for $S(\mathbf{q}, \omega)$ within the spinnematic phase of the quantum spin-ice model are shown in Fig. 5(a). Further details of the calculation of dynamical properties can be found in Appendix F.

\section{APPENDIX D: DEFINITIONS OF LOCAL ORDER-PARAMETER FIELDS}

The definitions of the local order-parameter fields $\mathbf{m}_{\lambda}$ that appear in the theory of the spin liquid $\mathrm{SL}_{\perp}$ (Sec. III) are given in Table I.

TABLE I. Order-parameter fields $\mathbf{m}_{\lambda}$, derived from irreducible representations (irreps) of the tetrahedral point group $\mathrm{T}_{d}$. Spin components $\mathrm{S}_{i}=\left(\mathrm{S}_{i}^{x}, \mathrm{~S}_{i}^{y}, \mathrm{~S}_{i}^{z}\right)$ are written in the local frame of the magnetic ions; see Appendix A for a definition of this coordinate frame. The convention for the labeling of the spins

\begin{tabular}{|c|c|}
\hline & Definition in terms of spins within tetrahedron \\
\hline$n_{\mathrm{A}_{2}}$ & $\frac{1}{2}\left(\mathrm{~S}_{0}^{z}+\mathrm{S}_{1}^{z}+\mathrm{S}_{2}^{z}+\mathrm{S}_{3}^{z}\right)$ \\
\hline $\mathbf{n}_{\mathrm{E}}$ & $\frac{1}{2}\left(\begin{array}{l}S_{0}^{x}+S_{1}^{x}+S_{2}^{x}+S_{3}^{x} \\
S_{0}^{y}+S_{1}^{y}+S_{2}^{y}+S_{3}^{y}\end{array}\right)$ \\
\hline $\mathbf{m}_{\mathrm{T}_{1, \mathrm{cec}}}$ & $\frac{1}{2}\left(\begin{array}{l}S_{0}^{z}+S_{1}^{z}-S_{2}^{z}-S_{3}^{z} \\
S_{0}^{z}-S_{1}^{z}+S_{2}^{z}-S_{3}^{z} \\
S_{0}^{z}-S_{1}^{z}-S_{2}^{z}+S_{3}^{z}\end{array}\right)$ \\
\hline $\mathbf{m}_{\mathrm{T}_{1, \mathrm{planar}}}$ & $\left(\begin{array}{c}\frac{1}{2}\left(\mathbf{S}_{0}^{x}+\mathbf{S}_{1}^{x}-\mathbf{S}_{2}^{x}-\mathbf{S}_{3}^{x}\right) \\
\frac{1}{4}\left(-\mathbf{S}_{0}^{x}+\sqrt{3} \mathbf{S}_{0}^{y}+\mathbf{S}_{1}^{x}-\sqrt{3} \mathbf{S}_{1}^{y}-\mathbf{S}_{2}^{x}+\sqrt{3} \mathbf{S}_{2}^{y}+\mathbf{S}_{3}^{x}-\sqrt{3} \mathbf{S}_{3}^{y}\right) \\
\frac{1}{4}\left(-\mathbf{S}_{0}^{x}-\sqrt{3} \mathbf{S}_{0}^{y}+\mathbf{S}_{1}^{x}+\sqrt{3} \mathbf{S}_{1}^{y}+\mathbf{S}_{2}^{x}+\sqrt{3} \mathbf{S}_{2}^{y}-\mathbf{S}_{3}^{x}-\sqrt{3} \mathbf{S}_{3}^{y}\right)\end{array}\right)$ \\
\hline $\mathrm{T}_{2}$ & $\left(\begin{array}{c}\frac{1}{2}\left(-S_{0}^{y}-S_{1}^{y}+S_{2}^{y}+S_{3}^{y}\right) \\
\frac{1}{4}\left(\sqrt{3} S_{0}^{x}+S_{0}^{y}-\sqrt{3} S_{1}^{x}-S_{1}^{y}+\sqrt{3} S_{2}^{x}+S_{2}^{y}-\sqrt{3} S_{3}^{x}-S_{3}^{y}\right) \\
\frac{1}{4}\left(-\sqrt{3} S_{0}^{x}+S_{0}^{y}+\sqrt{3} S_{1}^{x}-S_{1}^{y}+\sqrt{3} S_{2}^{x}-S_{2}^{y}-\sqrt{3} S_{3}^{x}+S_{3}^{y}\right)\end{array}\right)$ \\
\hline
\end{tabular}
with a tetrahedron is given in Appendix A.
Here we give the definitions in terms of the spins written in the local coordinate frame $\mathrm{S}_{i}$ (defined in Appendix A; cf. Ref. [49], where definitions are given in the global, crystal basis).

\section{APPENDIX E: NUMERICAL SIMULATION OF THE CORRELATIONS OF THE FLUX}

Values of the flux field $\mathbf{B}_{\mu}(\mathbf{r})$ are calculated for each tetrahedron $\mathbf{r}$ according to Eq. (8) and the definitions of $\mathbf{m}_{\lambda}$ given in Table I.

The tetrahedra of the pyrochlore lattice may be divided into two sets A and B. The centers of each set of tetrahedra each form a face-centered cubic (FCC) lattice.

To calculate $\mathbf{S}_{\mathbf{B}_{\mu}}^{\alpha \beta}$, we use Eq. (12), where $\mathbf{B}_{\mu}(\mathbf{q})$ is defined as the lattice Fourier transform of $\mathbf{B}_{\mu}(\mathbf{r})$ over only the $A$ sublattice of tetrahedra:

$$
\mathbf{B}_{\mu}(\mathbf{q})=\sqrt{\frac{1}{N_{\mathrm{ucc}}}} \sum_{\mathbf{r} \in \mathbf{r}_{A}} \exp \left(-i \mathbf{q} \cdot \mathbf{r}_{A}\right) \mathbf{B}_{\mu}(\mathbf{r}),
$$

where $N_{\text {u.c. }}$ is the number of unit cells in the system.

Simulations are carried out using local spin updates, augmented by overrelaxation, within a parallel tempering scheme with 300 temperatures distributed on a log scale between $T=0.003 J_{\mathrm{zz}}$ and $T=0.1 J_{\mathrm{zz}}$. Thermalization is accomplished through a process of simulated annealing, with $10^{4}$ Monte Carlo steps (MCs) of annealing from high temperature to temperature $T$, followed by $10^{4} \mathrm{MCs}$ of thermalization at temperature $T$, and $10^{5} \mathrm{MCs}$ of measurements at temperature $T$. Spin configurations are sampled every $100 \mathrm{MCs}$ during the measurements, giving an ensemble of 1000 samples.

\section{APPENDIX F: DYNAMICS OF EXCITATIONS IN THE SPIN-NEMATIC PHASE}

To study the Goldstone mode associated to the development of spin-nematic order, we calculate the dynamical correlation function

$$
\chi_{Q_{\perp}^{\text {site }}}(\mathbf{q}, \omega)=\left\langle\left|\delta \mathbf{Q}_{\perp}^{\text {site }}(\mathbf{q}, \omega)\right|^{2}\right\rangle,
$$

where fluctuations of spin-nematic order are given by

$$
\begin{aligned}
\delta \mathbf{Q}_{\perp}^{\text {site }}(\mathbf{q}, \omega) & =\sum_{i} \int d t\left[\mathbf{Q}_{\perp}^{\text {site }}\left(\mathbf{r}_{i}, t\right)-\overline{\mathbf{Q}}_{\perp}^{\text {site }}(t)\right] e^{i \omega t} e^{i \mathbf{q} \cdot \mathbf{r}_{i}} \\
\overline{\mathbf{Q}}_{\perp}^{\text {site }}(t) & =\frac{1}{N} \sum_{i} \mathbf{Q}_{\perp}^{\text {site }}\left(\mathbf{r}_{i}, t\right)
\end{aligned}
$$

and the order parameter $\mathbf{Q}_{\perp}^{\text {site }}\left(\mathbf{r}_{i}, t\right)$ is defined through Eq. (B2).

$\chi_{Q_{\perp}^{\text {site }}}(q, \omega)$ is calculated numerically from 200 sample configurations extracted from Monte Carlo simulations on 
(a) Dispersion relation of the Goldstone mode

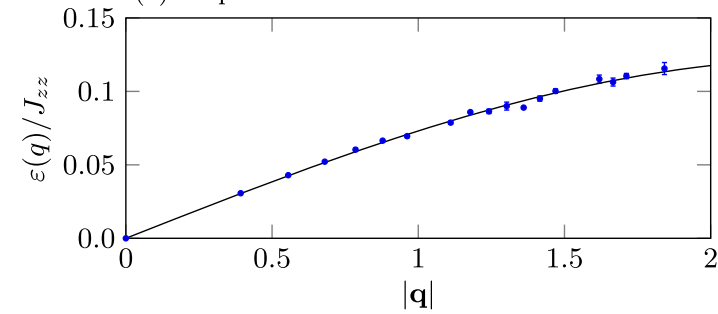

(b) Intensity of the quadrupolar waves

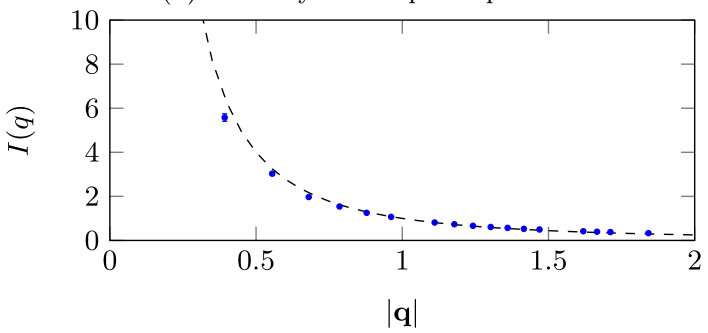

FIG. 8. Dispersion and intensity of the Goldstone mode in the phase with hidden spin-nematic order, as shown in Fig. 5(b). (a) Dispersion $\varepsilon(q)$ of low-energy peak in $\chi_{Q_{\perp}}(\mathbf{q}, \omega)$, showing the expected behavior $\varepsilon(q)=q$ at small $q$. (b) Intensity $I(q)$ of the peak as a function of momentum $q$. The dashed line shows the expected behavior at finite temperature, $I(q) \propto 1 / q^{2}$. Results are taken from molecular-dynamics simulations of a cluster of $N=$ 65536 spins, for $J_{ \pm} / J_{\mathrm{zz}}=-1.0, T=0.002 J_{\mathrm{zz}}$. Momentum $q$ is measured relative to $\mathbf{q}=(0,0,0)$.

a system of linear size $L=16$. We use 20000 steps for the simulated annealing spaced by ten Monte Carlo steps between each simulated annealing step. The other parameters for the thermalization and parallel tempering are identical to the parameters used to calculate the phase diagram (Appendix B).

The ensemble of configurations obtained from Monte Carlo is then evolved in time according to the equation of motion,

$$
\frac{d \mathrm{~S}_{i}}{d t}=\mathrm{S}_{i}(t) \times \mathrm{H}_{i}(t)
$$

where

$$
\mathrm{H}_{i}(t)=\sum_{j \in \mathrm{nn} i} \mathrm{~J}_{i j} \cdot \mathrm{S}_{j}(t)
$$

is the effective exchange field acting on site $i, J_{i j}$ is the anisotropic exchange interaction tensor, and the sum in Eq. (F4) runs over the neighbors of $i$. The numerical integration of this nonlinear equation of motion proceeds as described in Ref. [77].

In Fig. 8, we plot the dispersion of the Goldstone mode found in MD simulations of $\mathcal{H}_{\mathrm{XXZ}}$ within the spin-nematic phase for $J_{ \pm} / J_{\mathrm{zz}}=-1$. The dispersion is extracted from the position of the low-energy dispersing peak in $\chi_{Q_{\perp}}(\mathbf{q}, \omega)$, as shown in the inset in Fig. 5(b).
[1] P. W. Anderson, Resonating Valence Bonds: A New Kind of Insulator?, Mater. Res. Bull. 8, 153 (1973).

[2] P. A. Lee, An End to the Drought of Quantum Spin Liquids, Science 321, 1306 (2008).

[3] L. Balents, Spin Liquids in Frustrated Magnets, Nature (London) 464, 199 (2010).

[4] J. S. Gardner, M. J. P. Gingras, and J. E. Greedan, Magnetic Pyrochlore Oxides, Rev. Mod. Phys. 82, 53 (2010).

[5] S. T. Bramwell and M. J. P. Gingras, Spin Ice State in Frustrated Magnetic Pyrochlore Materials, Science 294, 1495 (2001).

[6] C. Castelnovo, R. Moessner, and S. L. Sondhi, Spin Ice, Fractionalization, and Topological Order, Annu. Rev. Condens. Matter Phys. 3, 35 (2012).

[7] S. H. Curnoe, Quantum Spin Configurations in $\mathrm{Tb}_{2} \mathrm{Ti}_{2} \mathrm{O}_{7}$, Phys. Rev. B 75, 212404 (2007).

[8] H. R. Molavian, M. J. P. Gingras, and B. Canals, Dynamically Induced Frustration as a Route to a Quantum Spin Ice State in $\mathrm{Tb}_{2} \mathrm{Ti}_{2} \mathrm{O}_{7}$ via Virtual Crystal Field Excitations and Quantum Many-Body Effects, Phys. Rev. Lett. 98, 157204 (2007).

[9] S. Onoda and Y. Tanaka, Quantum Melting of Spin Ice: Emergent Cooperative Quadrupole and Chirality, Phys. Rev. Lett. 105, 047201 (2010).

[10] S. Onoda and Y. Tanaka, Quantum Fluctuations in the Effective Pseudospin- $\frac{1}{2}$ Model for Magnetic Pyrochlore Oxides, Phys. Rev. B 83, 094411 (2011).

[11] K. A. Ross, L. Savary, B. D. Gaulin, and L. Balents, Quantum Excitations in Quantum Spin Ice, Phys. Rev. X 1, 021002 (2011).

[12] M. J. P. Gingras and P. A. McClarty, Quantum Spin Ice: A Search for Gapless Quantum Spin Liquids in Pyrochlore Magnets, Rep. Prog. Phys. 77, 056501 (2014).

[13] M. Hermele, M. P. A. Fisher, and L. Balents, Pyrochlore Photons: The U(1) Spin Liquid in a $S=\frac{1}{2}$ ThreeDimensional Frustrated Magnet, Phys. Rev. B 69, 064404 (2004).

[14] A. Banerjee, S. V. Isakov, K. Damle, and Y. B. Kim, Unusual Liquid State of Hard-Core Bosons on the Pyrochlore Lattice, Phys. Rev. Lett. 100, 047208 (2008).

[15] N. Shannon, O. Sikora, F. Pollmann, K. Penc, and P. Fulde, Quantum Ice: A Quantum Monte Carlo Study, Phys. Rev. Lett. 108, 067204 (2012).

[16] O. Benton, O. Sikora, and N. Shannon, Seeing the Light: Experimental Signatures of Emergent Electromagnetism in a Quantum Spin Ice, Phys. Rev. B 86, 075154 (2012).

[17] L. Savary and L. Balents, Coulombic Quantum Liquids in Spin-1/2 Pyrochlores, Phys. Rev. Lett. 108, 037202 (2012).

[18] S. B. Lee, S. Onoda, and L. Balents, Generic Quantum Spin Ice, Phys. Rev. B 86, 104412 (2012).

[19] Z. Hao, A. G. R. Day, and M. J. P. Gingras, Bosonic ManyBody Theory of Quantum Spin Ice, Phys. Rev. B 90, 214430 (2014).

[20] P. A. McClarty, O. Sikora, R. Moessner, K. Penc, F. Pollmann, and N. Shannon, Chain-Based Order and Quantum Spin Liquids in Dipolar Spin Ice, Phys. Rev. B 92, 094418 (2015).

[21] Y. Kato and S. Onoda, Numerical Evidence of Quantum Melting of Spin Ice: Quantum-to-Classical Crossover, Phys. Rev. Lett. 115, 077202 (2015). 
[22] G. Chen, "Magnetic Monopole" Condensation of the Pyrochlore Ice U(1) Quantum Spin Liquid: Application to $\mathrm{Pr}_{2} \mathrm{Ir}_{2} \mathrm{O}_{7}$ and $\mathrm{Yb}_{2} \mathrm{Ti}_{2} \mathrm{O}_{7}$, Phys. Rev. B 94, 205107 (2016).

[23] L. Savary and L. Balents, Disorder-Induced Quantum Spin Liquid in Spin Ice Pyrochlores, Phys. Rev. Lett. 118, 087203 (2017).

[24] G. Chen, Dirac's "Magnetic Monopoles" in Pyrochlore Ice U(1) Spin Liquids: Spectrum and Classification, Phys. Rev. B 96, 195127 (2017).

[25] C.-J. Huang, Y. Deng, Y. Wan, and Z. Y. Meng, Dynamics of Topological Excitations in a Model Quantum Spin Ice, arXiv:1707.00099.

[26] H. D. Zhou, C. R. Wiebe, J. A. Janik, L. Balicas, Y. J. Yo, Y. Qiu, J. R. D. Copley, and J. S. Gardner, Dynamic Spin Ice: $\operatorname{Pr}_{2} \mathrm{Sn}_{2} \mathrm{O}_{7}$, Phys. Rev. Lett. 101, 227204 (2008).

[27] L. J. Chang, S. Onoda, Y. Su, Y. J. Kao, K. D. Tsuei, Y. Yasui, K. Kakurai, and M. R. Lees, Higgs Transition from a Magnetic Coulomb Liquid to a Ferromagnet in $\mathrm{Yb}_{2} \mathrm{Ti}_{2} \mathrm{O}_{7}$, Nat. Commun. 3, 992 (2012).

[28] T. Fennell, M. Kenzelmann, B. Roessli, M. K. Haas, and R. J. Cava, Power-Law Spin Correlations in the Pyrochlore Antiferromagnet $\mathrm{Tb}_{2} \mathrm{Ti}_{2} \mathrm{O}_{7}$, Phys. Rev. Lett. 109, 017201 (2012).

[29] K. Kimura, S. Nakatsuji, J.-J. Wen, C. Broholm, M. B. Stone, E. Nishibori, and H. Sawa, Quantum Fluctuations in Spin-Ice-Like $\operatorname{Pr}_{2} \mathrm{Zr}_{2} \mathrm{O}_{7}$, Nat. Commun. 4, 1934 (2013).

[30] R. Sibille, E. Lhotel, V. Pomjakushin, C. Baines, T. Fennell, and M. Kenzelmann, Candidate Quantum Spin Liquid in the $\mathrm{Ce}^{3+}$ Pyrochlore Stannate $\mathrm{Ce}_{2} \mathrm{Sn}_{2} \mathrm{O}_{7}$, Phys. Rev. Lett. 115, 097202 (2015).

[31] R. Sibille, E. Lhotel, M. C. Hatnean, G. Balakrishnan, B. Fåk, N. Gauthier, T. Fennell, and M. Kenzelmann, Candidate Quantum Spin Ice in the Pyrochlore $\operatorname{Pr}_{2} \mathrm{Hf}_{2} \mathrm{O}_{7}$, Phys. Rev. B 94, 024436 (2016).

[32] V. K. Anand, L. Opherden, J. Xu, D. T. Adroja, A. T. M. N. Islam, T. Herrmannsdörfer, J. Hornung, R. Schönemann, M. Uhlarz, H. C. Walker, N. Casati, and B. Lake, Physical Properties of the Candidate Quantum Spin-Ice System $\mathrm{Pr}_{2} \mathrm{Hf}_{2} \mathrm{O}_{7}$, Phys. Rev. B 94, 144415 (2016).

[33] J.-J. Wen, S. M. Koohpayeh, K. A. Ross, B. A. Trump, T. M. McQueen, K. Kimura, S. Nakatsuji, Y. Qiu, D. M. Pajerowski, J. R. D. Copley, and C. L. Broholm, Disordered Route to the Coulomb Quantum Spin Liquid: Random Transverse Fields on Spin Ice in $\mathrm{Pr}_{2} \mathrm{Zr}_{2} \mathrm{O}_{7}$, Phys. Rev. Lett. 118, 107206 (2017).

[34] R. Sibille, N. Gauthier, H. Yan, M. C. Hatnean, J. Ollivier, B. Winn, G. Balakrishnan, M. Kenzelmann, N. Shannon, and T. Fennell, Experimental Signatures of Emergent Quantum Electrodynamics in a Quantum Spin Ice, arXiv:1706.03604.

[35] P. Dalmas de Réotier, A. Yaouanc, L. Keller, A. Cervellino, B. Roessli, C. Baines, A. Forget, C. Vaju, P. C. M. Gubbens, A. Amato, and P. J. C. King, Spin Dynamics and Magnetic Order in Magnetically Frustrated $\mathrm{Tb}_{2} \mathrm{Sn}_{2} \mathrm{O}_{7}$, Phys. Rev. Lett. 96, 127202 (2006).

[36] Z. L. Dun, E. S. Choi, H. D. Zhou, A. M. Hallas, H. J. Silverstein, Y. Qiu, J. R. D. Copley, J. S. Gardner, and
C. R. Wiebe, $\mathrm{Yb}_{2} \mathrm{Sn}_{2} \mathrm{O}_{7}$ : A Magnetic Coulomb Liquid at a Quantum Critical Point, Phys. Rev. B 87, 134408 (2013).

[37] A. Yaouanc, P. Dalmas de Réotier, P. Bonville, J. A. Hodges, V. Glazkov, L. Keller, V. Sikolenko, M. Bartkowiak, A. Amato, C. Baines, P. J. C. King, P. C. M. Gubbens, and A. Forget, Dynamical Splayed Ferromagnetic Ground State in the Quantum Spin Ice $\mathrm{Yb}_{2} \mathrm{Sn}_{2} \mathrm{O}_{7}$, Phys. Rev. Lett. 110, 127207 (2013).

[38] T. Taniguchi, H. Kadowaki, H. Takatsu, B. Fåk, J. Ollivier, T. Yamazaki, T. J. Sato, H. Yoshizawa, Y. Shimura, T. Sakakibara, T. Hong, K. Goto, L. R. Yaraskavitch, and J. B. Kycia, Long-Range Order and Spin-Liquid States of Polycrystalline $\mathrm{Tb}_{2+x} \mathrm{Ti}_{2-x} \mathrm{O}_{7+y}$, Phys. Rev. B 87, 060408 (2013).

[39] A. M. Hallas, J. G. Cheng, A. M. Arevalo-Lopez, H. J. Silverstein, Y. Su, P. M. Sarte, H. D. Zhou, E. S. Choi, J. P. Attfield, G. M. Luke, and C. R. Wiebe, Incipient Ferromagnetism in $\mathrm{Tb}_{2} \mathrm{Ge}_{2} \mathrm{O}_{7}$ : Application of Chemical Pressure to the Enigmatic Spin-Liquid Compound $\mathrm{Tb}_{2} \mathrm{Ti}_{2} \mathrm{O}_{7}$, Phys. Rev. Lett. 113, 267205 (2014).

[40] A. M. Hallas, A. M. Arevalo-Lopez, A. Z. Sharma, T. Munsie, J. P. Attfield, C. R. Wiebe, and G. M. Luke, Magnetic Frustration in Lead Pyrochlores, Phys. Rev. B 91, 104417 (2015).

[41] A. M. Hallas, J. Gaudet, N. P. Butch, M. Tachibana, R. S. Freitas, G. M. Luke, C. R. Wiebe, and B. D. Gaulin, Universal Dynamic Magnetism in Yb Pyrochlores with Disparate Ground States, Phys. Rev. B 93, 100403 (2016).

[42] S. Petit, E. Lhotel, B. Canals, M. C. Hatnean, J. Ollivier, H. Mutka, E. Ressouche, A. R. Wildes, M. R. Lees, and G. Balakrishnan, Observation of Magnetic Fragmentation in Spin Ice, Nat. Phys. 12, 746 (2016).

[43] S. Petit, E. Lhotel, S. Guitteny, O. Florea, J. Robert, P. Bonville, I. Mirebeau, J. Ollivier, H. Mutka, E. Ressouche, C. Decorse, M. Ciomaga Hatnean, and G. Balakrishnan, Antiferroquadrupolar Correlations in the Quantum Spin Ice Candidate $\mathrm{Pr}_{2} \mathrm{Zr}_{2} \mathrm{O}_{7}$, Phys. Rev. B 94, 165153 (2016).

[44] H. Takatsu, S. Onoda, S. Kittaka, A. Kasahara, Y. Kono, T. Sakakibara, Y. Kato, B. Fåk, J. Ollivier, J. W. Lynn, T. Taniguchi, M. Wakita, and H. Kadowaki, Quadrupole Order in the Frustrated Pyrochlore $\mathrm{Tb}_{2+x} \mathrm{Ti}_{2-x} \mathrm{O}_{7+y}$, Phys. Rev. Lett. 116, 217201 (2016).

[45] A. M. Hallas, J. Gaudet, and B. D. Gaulin, Experimental Insights into Ground State Selection of Quantum XY Pyrochlores, arXiv:1708.01312 [Annu. Rev. Condens. Matter Phys. (to be published)].

[46] T. Fennell, P. P. Deen, A. R. Wildes, K. Schmalzl, D. Prabhakaran, A. T. Boothroyd, R. J. Aldus, D. F. McMorrow, and S. T. Bramwell, Magnetic Coulomb Phase in the Spin Ice $\mathrm{Ho}_{2} \mathrm{Ti}_{2} \mathrm{O}_{7}$, Science 326, 415 (2009).

[47] S. Onoda, Effective Quantum Pseudospin-1/2 Model for Yb Pyrochlore Oxides, J. Phys. Conf. Ser. 320, 012065 (2011).

[48] A. W. C. Wong, Z. Hao, and M. J. P. Gingras, Ground State Phase Diagram of Generic XY Pyrochlore Magnets with Quantum Fluctuations, Phys. Rev. B 88, 144402 (2013).

[49] H. Yan, O. Benton, L. Jaubert, and N. Shannon, Theory of Multiple-Phase Competition in Pyrochlore Magnets with Anisotropic Exchange with Application to $\mathrm{Yb}_{2} \mathrm{Ti}_{2} \mathrm{O}_{7}, \mathrm{Er}_{2} \mathrm{Ti}_{2} \mathrm{O}_{7}$, and $\mathrm{Er}_{2} \mathrm{Sn}_{2} \mathrm{O}_{7}$, Phys. Rev. B 95, 094422 (2017). 
[50] O. Benton, Classical and Quantum Spin Liquids on the Pyrochlore Lattice, Ph.D. thesis, University of Bristol, 2015.

[51] Y.-P. Huang, G. Chen, and M. Hermele, Quantum Spin Ices and Topological Phases from Dipolar-Octupolar Doublets on the Pyrochlore Lattice, Phys. Rev. Lett. 112, 167203 (2014).

[52] G. Chen, Spectral Periodicity of the Spinon Continuum in Quantum Spin Ice, Phys. Rev. B 96, 085136 (2017).

[53] R. Moessner and J. T. Chalker, Properties of a Classical Spin Liquid: The Heisenberg Pyrochlore Antiferromagnet, Phys. Rev. Lett. 80, 2929 (1998).

[54] R. Moessner and J. T. Chalker, Low-Temperature Properties of Classical Geometrically Frustrated Antiferromagnets, Phys. Rev. B 58, 12049 (1998).

[55] S. V. Isakov, K. Gregor, R. Moessner, and S. L. Sondhi, Dipolar Spin Correlations in Classical Pyrochlore Magnets, Phys. Rev. Lett. 93, 167204 (2004).

[56] C. L. Henley, Power-Law Spin Correlations in Pyrochlore Antiferromagnets, Phys. Rev. B 71, 014424 (2005).

[57] B. Canals and C. Lacroix, Pyrochlore Antiferromagnet: A Three-Dimensional Quantum Spin Liquid, Phys. Rev. Lett. 80, 2933 (1998).

[58] B. Canals and C. Lacroix, Quantum Spin Liquid: The Heisenberg Antiferromagnet on the Three-Dimensional Pyrochlore Lattice, Phys. Rev. B 61, 1149 (2000).

[59] F. J. Burnell, S. Chakravarty, and S. L. Sondhi, Monopole Flux State on the Pyrochlore Lattice, Phys. Rev. B 79, 144432 (2009).

[60] Y. Huang, K. Chen, Y. Deng, N. Prokof'ev, and B. Svistunov, Spin-Ice State of the Quantum Heisenberg Antiferromagnet on the Pyrochlore Lattice, Phys. Rev. Lett. 116, 177203 (2016).

[61] O. Benton (unpublished).

[62] M. Taillefumier, O. Benton, and N. Shannon (to be published).

[63] L. D. C. Jaubert, M. J. Harris, T. Fennell, R. G. Melko, S. T. Bramwell, and P. C. W. Holdsworth, Topological-Sector Fluctuations and Curie-Law Crossover in Spin Ice, Phys. Rev. X 3, 011014 (2013).

[64] P. H. Conlon and J. T. Chalker, Spin Dynamics in Pyrochlore Heisenberg Antiferromagnets, Phys. Rev. Lett. 102, 237206 (2009).

[65] C. L. Henley, The "Coulomb Phase" in Frustrated Systems, Annu. Rev. Condens. Matter Phys. 1, 179 (2010).

[66] A. F. Andreev and I. A. Grishchuk, Spin Nematics, JETP 87, 467 (1984).

[67] A. V. Chubukov, Chiral, Nematic, and Dimer States in Quantum Spin Chains, Phys. Rev. B 44, 4693 (1991).

[68] N. Shannon, T. Momoi, and P. Sindzingre, Nematic Order in Square Lattice Frustrated Ferromagnets, Phys. Rev. Lett. 96, 027213 (2006).

[69] N. Shannon, K. Penc, and Y. Motome, Nematic, VectorMultipole, and Plateau-Liquid States in the Classical O(3) Pyrochlore Antiferromagnet with Biquadratic Interactions in Applied Magnetic Field, Phys. Rev. B 81, 184409 (2010).

[70] A. Smerald, H. T. Ueda, and N. Shannon, Theory of Inelastic Neutron Scattering in a Field-Induced SpinNematic State, Phys. Rev. B 91, 174402 (2015).
[71] S. V. Isakov, R. Moessner, and S. L. Sondhi, Why Spin Ice Obeys the Ice Rules, Phys. Rev. Lett. 95, 217201 (2005).

[72] O. Benton, L. D. C. Jaubert, H. Yan, and N. Shannon, A Spin-Liquid with Pinch-Line Singularities on the Pyrochlore Lattice, Nat. Commun. 7, 11572 (2016).

[73] P. A. McClarty, S. H. Curnoe, and M. J. P. Gingras, Energetic Selection of Ordered States in a Model of the $\mathrm{Er}_{2} \mathrm{Ti}_{2} \mathrm{O}_{7}$ Frustrated Pyrochlore XY Antiferromagnet, J. Phys. Conf. Ser. 145, 012032 (2009).

[74] H. Yan, O. Benton, L. Jaubert, and N. Shannon, Living on the Edge: Ground-State Selection in Quantum Spin-Ice Pyrochlores, arXiv:1311.3501.

[75] L. D. C. Jaubert, O. Benton, J. G. Rau, J. Oitmaa, R. R. P. Singh, N. Shannon, and M. J. P. Gingras, Are Multiphase Competition and Order by Disorder the Keys to Understanding $\mathrm{Yb}_{2} \mathrm{Ti}_{2} \mathrm{O}_{7}$ ?, Phys. Rev. Lett. 115, 267208 (2015).

[76] S. E. Palmer and J. T. Chalker, Order Induced by Dipolar Interactions in a Geometrically Frustrated Antiferromagnet, Phys. Rev. B 62, 488 (2000).

[77] M. Taillefumier, J. Robert, C. L. Henley, R. Moessner, and B. Canals, Semiclassical Spin Dynamics of the Antiferromagnetic Heisenberg Model on the Kagome Lattice, Phys. Rev. B 90, 064419 (2014).

[78] J. Knolle, D. L. Kovrizhin, J. T. Chalker, and R. Moessner, Dynamics of a Two-Dimensional Quantum Spin Liquid: Signatures of Emergent Majorana Fermions and Fluxes, Phys. Rev. Lett. 112, 207203 (2014).

[79] M. Punk, D. Chowdhury, and S. Sachdev, Topological Excitations and the Dynamic Structure Factor of Spin Liquids on the Kagome Lattice, Nat. Phys. 10, 289 (2014).

[80] S. Bieri, L. Messio, B. Bernu, and C. Lhuillier, Gapless Chiral Spin Liquid in a Kagome Heisenberg Model, Phys. Rev. B 92, 060407 (2015).

[81] T. H. Han, J. S. Helton, S. Chu, D. G. Nocera, J. A. Rodriguez-Rivera, C. Broholm, and Y. S. Lee, Fractionalized Excitations in the Spin-Liquid State of a KagomeLattice Antiferromagnet, Nature (London) 492, 406 (2012).

[82] J. A. M. Paddison, M. Daum, Z. L. Dun, G. Ehlers, Y. Liu, M. B. Stone, H. Zhou, and M. Mourigal, Continuous Excitations of the Triangular-Lattice Quantum Spin Liquid $\mathrm{YbMgGaO}_{4}$, Nat. Phys. 13, 117 (2017).

[83] L. Savary and L. Balents, Quantum Spin Liquids: A Review, Rep. Prog. Phys. 80, 016502 (2017).

[84] L. Savary and T. Senthil, Probing Hidden Orders with Resonant Inelastic X-Ray Scattering, arXiv:1506.04752.

[85] A. Smerald and N. Shannon, Theory of Spin Excitations in a Quantum Spin-Nematic State, Phys. Rev. B 88, 184430 (2013).

[86] O. A. Starykh and L. Balents, Excitations and Quasi-OneDimensionality in Field-Induced Nematic and Spin Density Wave States, Phys. Rev. B 89, 104407 (2014).

[87] A. Smerald and N. Shannon, Theory of $N M R 1 / T_{1}$ Relaxation in a Quantum Spin Nematic in an Applied Magnetic Field, Phys. Rev. B 93, 184419 (2016).

[88] H. Tsunetsugu and M. Arikawa, Spin Nematic Phase in $S=1$ Triangular Antiferromagnets, J. Phys. Soc. Jpn. 75, 083701 (2006). 
[89] A. Läuchli, F. Mila, and K. Penc, Quadrupolar Phases of the $S=1$ Bilinear-Biquadratic Heisenberg Model on the Triangular Lattice, Phys. Rev. Lett. 97, 087205 (2006).

[90] A. Völl and S. Wessel, Spin Dynamics of the BilinearBiquadratic $S=1$ Heisenberg Model on the Triangular Lattice: A Quantum Monte Carlo Study, Phys. Rev. B 91, 165128 (2015).

[91] H. Onishi, Magnetic Excitations of Spin Nematic State in Frustrated Ferromagnetic Chain, J. Phys. Soc. Jpn. 84, 083702 (2015).

[92] R. Shindou, S. Yunoki, and T. Momoi, Dynamical Spin Structure Factors of Quantum Spin Nematic States, Phys. Rev. B 87, 054429 (2013).

[93] S. Guitteny, J. Robert, P. Bonville, J. Ollivier, C. Decorse, P. Steffens, M. Boehm, H. Mutka, I. Mirebeau, and S. Petit, Anisotropic Propagating Excitations and Quadrupolar Effects in $\mathrm{Tb}_{2} \mathrm{Ti}_{2} \mathrm{O}_{7}$, Phys. Rev. Lett. 111, 087201 (2013).

[94] Y.-D. Li and G. Chen, Symmetry Enriched U(1) Topological Orders for Dipole-Octupole Doublets on a Pyrochlore Lattice, Phys. Rev. B 95, 041106 (2017).

[95] O. Benton, From Quantum Spin Liquid to Paramagnetic Ground States in Disordered Non-Kramers Pyrochlores, arXiv:1706.09238.

[96] N. Martin, P. Bonville, E. Lhotel, S. Guitteny, A. Wildes, C. Decorse, M. C. Hatnean, G. Balakrishnan, I. Mirebeau, and S. Petit, Disorder and Quantum Spin Ice, Phys. Rev. X 7, 041028 (2017)

[97] Z. L. Dun, M. Lee, E. S. Choi, A. M. Hallas, C. R. Wiebe, J. S. Gardner, E. Arrighi, R. S. Freitas, A. M. ArevaloLopez, J. P. Attfield, H. D. Zhou, and J. G. Cheng, Chemical Pressure Effects on Magnetism in the Quantum Spin Liquid Candidates $\mathrm{Yb}_{2} \mathrm{X}_{2} \mathrm{O}_{7}(\mathrm{X}=\mathrm{Sn}$, Ti, Ge), Phys. Rev. B 89, 064401 (2014).

[98] C. R. Wiebe and A. M. Hallas, Frustration under Pressure: Exotic Magnetism in New Pyrochlore Oxides, APL Mater. 3, 041519 (2015).

[99] K. Matsuhira, C. Sekine, C. Paulsen, and Y. Hinatsu, LowTemperature Magnetic Properties of the Geometrically Frustrated Pyrochlore $\operatorname{Pr}_{2} \mathrm{Sn}_{2} \mathrm{O}_{7}$, J. Magn. Magn. Mater. 272-276, E981 (2004).

[100] J. Robert, E. Lhotel, G. Remenyi, S. Sahling, I. Mirebeau, C. Decorse, B. Canals, and S. Petit, Spin Dynamics in the Presence of Competing Ferromagnetic and Antiferromagnetic Correlations in $\mathrm{Yb}_{2} \mathrm{Ti}_{2} \mathrm{O}_{7}$, Phys. Rev. B 92, 064425 (2015).

[101] J. D. Thompson, P. A. McClarty, D. Prabhakaran, I. Cabrera, T. Guidi, and R. Coldea, Quasiparticle Breakdown and Spin Hamiltonian of the Frustrated Quantum Pyrochlore $\mathrm{Yb}_{2} \mathrm{Ti}_{2} \mathrm{O}_{7}$ in a Magnetic Field, Phys. Rev. Lett. 119, 057203 (2017).

[102] K. A. Ross, J. P. C. Ruff, C. P. Adams, J. S. Gardner, H. A. Dabkowska, Y. Qiu, J. R. D. Copley, and B. D. Gaulin, Two-Dimensional Kagome Correlations and Field Induced Order in the Ferromagnetic XY Pyrochlore $\mathrm{Yb}_{2} \mathrm{Ti}_{2} \mathrm{O}_{7}$, Phys. Rev. Lett. 103, 227202 (2009).

[103] A. Maisuradze, P. Dalmas de Réotier, A. Yaouanc, A. Forget, C. Baines, and P. J. C. King, Anomalously Slow Spin Dynamics and Short-Range Correlations in the Quantum Spin Ice Systems $\mathrm{Yb}_{2} \mathrm{Ti}_{2} \mathrm{O}_{7}$ and $\mathrm{Yb}_{2} \mathrm{Sn}_{2} \mathrm{O}_{7}$, Phys. Rev. B 92, 094424 (2015).
[104] J. Gaudet, K. A. Ross, E. Kermarrec, N. P. Butch, G. Ehlers, H. A. Dabkowska, and B. D. Gaulin, Gapless Quantum Excitations from an Icelike Splayed Ferromagnetic Ground State in Stoichiometric $\mathrm{Yb}_{2} \mathrm{Ti}_{2} \mathrm{O}_{7}$, Phys. Rev. B 93, 064406 (2016).

[105] Y. Yasui, M. Soda, S. Iikubo, M. Ito, M. Sato, N. Hamaguchi, T. Matsushita, N. Wada, T. Takeuchi, N. Aso, and K. Kakurai, Ferromagnetic Transition of Pyrochlore Compound $\mathrm{Yb}_{2} \mathrm{Ti}_{2} \mathrm{O}_{7}$, J. Phys. Soc. Jpn. 72, 3014 (2003).

[106] E. Lhotel, S. R. Giblin, M. R. Lees, G. Balakrishnan, L. J. Chang, and Y. Yasui, First-Order Magnetic Transition in $\mathrm{Yb}_{2} \mathrm{Ti}_{2} \mathrm{O}_{7}$, Phys. Rev. B 89, 224419 (2014).

[107] A. M. Hallas, J. Gaudet, M. N. Wilson, T. J. Munsie, A. A. Aczel, M. B. Stone, R. S. Freitas, A. M. Arevalo-Lopez, J. P. Attfield, M. Tachibana, C. R. Wiebe, G. M. Luke, and B. D. Gaulin, XY Antiferromagnetic Ground State in the Effective $S=\frac{1}{2}$ Pyrochlore $\mathrm{Yb}_{2} \mathrm{Ge}_{2} \mathrm{O}_{7}$, Phys. Rev. B 93, 104405 (2016).

[108] S. Powell, Ferromagnetic Coulomb Phase in Classical Spin Ice, Phys. Rev. B 91, 094431 (2015).

[109] M. E. Brooks-Bartlett, S. T. Banks, L. D C Jaubert, A. Harman-Clarke, and P. C W Holdsworth, MagneticMoment Fragmentation and Monopole Crystallization, Phys. Rev. X 4, 011007 (2014).

[110] O. Benton, Quantum Origins of Moment Fragmentation in $\mathrm{Nd}_{2} \mathrm{Zr}_{2} \mathrm{O}_{7}$, Phys. Rev. B 94, 104430 (2016).

[111] E. Lefrançois, V. Cathelin, E. Lhotel, J. Robert, P. Lejay, C. V. Colin, B. Canals, F. Damay, J. Ollivier, B. Fåk, L. C. Chapon, R. Ballou, and V. Simonet, Fragmentation in Spin Ice from Magnetic Charge Injection, Nat. Commun. 8, 209 (2017).

[112] K. A. Ross, J. W. Krizan, J. A. Rodriguez-Rivera, R. J. Cava, and C. L. Broholm, Static and Dynamic XY-like Short-Range Order in a Frustrated Magnet with Exchange Disorder, Phys. Rev. B 93, 014433 (2016).

[113] K. A. Ross, J. M. Brown, R. J. Cava, J. W. Krizan, S. E. Nagler, J. A. Rodriguez-Rivera, and M. B. Stone, SingleIon Properties of the $S_{\mathrm{eff}}=\frac{1}{2} X Y$ Antiferromagnetic Pyrochlores $\mathrm{Na}^{\prime} \mathrm{Co}_{2} \mathrm{~F}_{7}\left(A^{\prime}=\mathrm{Ca}^{2+}, \mathrm{Sr}^{2+}\right)$, Phys. Rev. B 95, 144414 (2017).

[114] K. A. Ross, Th. Proffen, H. A. Dabkowska, J. A. Quilliam, L. R. Yaraskavitch, J. B. Kycia, and B. D. Gaulin, Lightly Stuffed Pyrochlore Structure of Single-Crystalline $\mathrm{Yb}_{2} \mathrm{Ti}_{2} \mathrm{O}_{7}$ Grown by the Optical Floating Zone Technique, Phys. Rev. B 86, 174424 (2012).

[115] A. Mostaed, G. Balakrishnan, M. R. Lees, Y. Yasui, L. J. Chang, and R. Beanland, Atomic Structure Study of the Pyrochlore $\mathrm{Yb}_{2} \mathrm{Ti}_{2} \mathrm{O}_{7}$ and Its Relationship with LowTemperature Magnetic Order, Phys. Rev. B 95, 094431 (2017).

[116] E. Kermarrec, A. Zorko, F. Bert, R. H. Colman, B. Koteswararao, F. Bouquet, P. Bonville, A. Hillier, A. Amato, J. van Tol, A. Ozarowski, A. S. Wills, and P. Mendels, Spin Dynamics and Disorder Effects in the $S=\frac{1}{2}$ Kagome Heisenberg Spin-Liquid Phase of Kapellasite, Phys. Rev. B 90, 205103 (2014).

[117] T.-H. Han, M. R. Norman, J.-J. Wen, J. A. RodriguezRivera, J. S. Helton, C. Broholm, and Y. S. Lee, Correlated Impurities and Intrinsic Spin-Liquid Physics in the 
Kagome Material Herbertsmithite, Phys. Rev. B 94, 060409 (2016).

[118] M. R. Norman, Colloquium: Herbertsmithite and the Search for the Quantum Spin Liquid, Rev. Mod. Phys. 88, 041002 (2016).

[119] Y. Li, D. Adroja, R. I. Bewley, D. Voneshen, A. A. Tsirlin, P. Gegenwart, and Q. Zhang, Crystalline Electric-Field Randomness in the Triangular Lattice Spin-Liquid $\mathrm{YbMgGaO}_{4}$, Phys. Rev. Lett. 118, 107202 (2017).

[120] L. Bellier-Castella, M. J. P. Gingras, P. C. W. Holdsworth, and R. Moessner, Frustrated Order by Disorder: The
Pyrochlore Anti-Ferromagnet with Bond Disorder, Can. J. Phys. 79, 1365 (2001).

[121] T. E. Saunders and J. T. Chalker, Spin Freezing in Geometrically Frustrated Antiferromagnets with Weak Disorder, Phys. Rev. Lett. 98, 157201 (2007).

[122] H. Tsunetsugu, Antiferromagnetic Quantum Spins on the Pyrochlore Lattice, J. Phys. Soc. Jpn. 70, 640 (2001).

[123] H. T. Ueda, Y. Akagi, and N. Shannon, Quantum Solitons with Emergent Interactions in a Model of Cold Atoms on the Triangular Lattice, Phys. Rev. A 93, 021606 (2016).

[124] R. R. P. Singh and J. Oitmaa (unpublished). 